\title{
SINGLE-CHANGE CIRCULAR COVERING DESIGNS
}

\author{
John P. McSorley \\ Department of Mathematics, Southern Illinois University, \\ Carbondale. IL 62901-4408. jmcsorley@math.siu.edu
}

Keywords Single-change, circular, covering, design, tight.

\begin{abstract}
A single-change circular covering design (scccd) based on the set $[v]=\{1, \ldots, v\}$ with block size $k$ is an ordered collection of $b$ blocks, $\mathcal{B}=\left\{B_{1}, \ldots, B_{b}\right\}$, each $B_{i} \subset[v]$, which obey: (1) each block differs from the previous block by a single element, as does the last from the first, and, (2) every pair of $[v]$ is covered by some $B_{i}$. The object is to minimize $b$ for a fixed $v$ and $k$. We present some minimal constructions of scccds for arbitrary $v$ when $k=2$ and 3 , and for arbitrary $k$ when $k+1 \leq v \leq 2 k$. Tight designs are those in which each pair is covered exactly once. Start-Finish arrays are used to construct tight designs when $v>2 k$; there are 2 non-isomorphic tight designs with $(v, k)=(9,4)$, and 12 with $(v, k)=(10,4)$. Some non-existence results for tight designs, and standardized, element-regular, perfect, and column-regular designs are also considered.
\end{abstract}

\section{Definitions; notation; examples}

A single-change circular covering design based on the set $[v]=\{1, \ldots, v\}$ with block size $k$ is an ordered collection of $b$ blocks, $\mathcal{B}=\left\{B_{1}, \ldots, B_{b}\right\}$, each an unordered subset of $k$ distinct elements from $[v]$, which obey:

(1) each block differs from the previous block by a single element, i.e., $\left|B_{i-1} \cap B_{i}\right|=k-1$ for $i=2, \ldots, b$; and the last block, $B_{b}$, differs from the first, $B_{1}$, by a single element, i.e., $\left|B_{b} \cap B_{1}\right|=k-1$; and

(2) every (unordered) pair $\{x, y\}$ of $[v]$, with $x \neq y$, can be written as $\left\{e_{i}, z\right\}$ where $e_{i} \in B_{i} \backslash B_{i-1}$ and $z \in B_{i}$ for some $i=2, \ldots, b$, or as $\left\{e_{1}, z\right\}$ where $e_{1} \in B_{1} \backslash B_{b}$ and $z \in B_{1}$.

For $i=2, \ldots, b$ we say that element $e_{i}$ is introduced in block $B_{i}$, and the pairs $\left\{e_{i}, z\right\}$ where $z \in B_{i}$ are covered by $B_{i}$. Similarly, $e_{1}$ is introduced in $B_{1}$ and pairs $\left\{e_{1}, z\right\}$ where $z \in B_{1}$ are covered by $B_{1}$. We also say that a pair is covered by $\mathcal{B}$ if it is covered by some block in $\mathcal{B}$.

A single-change circular covering design is simply a single-change covering design (see Wallis, Yucas, and Zhang []], and Preece, Constable, Zhang, Yucas, Wallis, McSorley, and Phillips [2] ) in which a 'single-change' is also required between $B_{b}$ and $B_{1}$. 
We denote a single-change circular covering design by scccd; and a scccd based on $[v]$ with block size $k$ by $\operatorname{scccd}(v, k)$, or by $\operatorname{scccd}(v, k, b)$ if we wish to mention that it contains $b$ blocks. For a fixed $v$ and $k$, where $k \geq 2$ and $v \geq k+1$, we denote by $b_{*}(v, k)$ the smallest $b$ for which there exists a $\operatorname{scccd}(v, k, b)$, and we call $\operatorname{such} a \operatorname{scccd}\left(v, k, b_{*}(v, k)\right)$ minimal. We write our designs vertically as in [ㅍ] .

Our first example is a $\operatorname{scccd}(6,3,8)$ labelled $\mathcal{E}$ and shown in Fig. 1(a). In $\mathcal{E}$ each pair, except $\{4,1\}$, is covered once; $\{4,1\}$ is covered twice, in $B_{5}$ and in $B_{8}$. This is an economical design, see $\S 5$.

\begin{tabular}{ccccc}
\multicolumn{9}{c}{ element } \\
blocks & introduced & pairs covered \\
$B_{i}$ & \multicolumn{1}{c}{$e_{i}$} & $\left\{e_{i}, z\right\}, z \in B_{i}$ \\
$B_{1}$ & 642 & 6 & $\{6,4\}$ & $\{6,2\}$ \\
$B_{2}$ & 632 & 3 & $\{3,6\}$ & $\{3,2\}$ \\
$B_{3}$ & 635 & 5 & $\{5,6\}$ & $\{5,3\}$ \\
$B_{4}$ & 631 & 1 & $\{1,6\}$ & $\{1,3\}$ \\
$B_{5}$ & 431 & 4 & $\{4,3\}$ & $\{4,1\}$ \\
$B_{6}$ & 451 & 5 & $\{5,4\}$ & $\{5,1\}$ \\
$B_{7}$ & 251 & 2 & $\{2,5\}$ & $\{2,1\}$ \\
$B_{8}$ & 241 & 4 & $\{4,2\}$ & $\{4,1\}$ \\
\multicolumn{5}{c}{ E an economical scccd $(6,3,8)$}
\end{tabular}

(a)
123

345

451

512

Fig. 1: Examples: $\mathcal{E}$ and $\mathcal{Y}_{3}$.

$\mathcal{Y}_{3}$, a tight $\operatorname{scccd}(5,3,5)$

(b)

As the ordering of the elements in a block is immaterial, we often (but not always) leave a block's unchanged elements in the same columns as in the previous block, see $\mathcal{E}$ in Fig. 1(a); such a representation of $\mathcal{E}$ is called column-strict. In block $B_{4}$ element 1 is introduced because $1 \notin B_{3}$ and element 6 is changed because $6 \notin B_{5}$.

Our second example is a $\operatorname{scccd}(5,3,5)$, see Fig. 1(b); we call this design $\mathcal{Y}_{3}$, see $\S 6$. Here the 10 pairs from the set $\{1,2,3,4,5\}$ are each covered exactly once, 2 per block; we call such a design tight, see $\S 5$.

We generally use the notation $\mathcal{B}=\left\{B_{1}, \ldots, B_{b}\right\}$ for an $\operatorname{arbitrary} \operatorname{scccd}(v, k, b)$ and $\mathcal{C}$ for an arbitrary tight $\operatorname{scccd}(v, k, b)$, often with $v>2 k$. 
The main object of this paper is to study scccds, with special interest in the function $b_{*}(v, k)$ and in tight designs. We will see that the structure and construction of these designs are somewhat different from those of the single-change covering designs of [ 4$]$ and [2]

\section{Requirements for $\mathcal{B}$ to form a single-change circular covering design}

Let $\mathcal{B}=\left\{B_{1}, \ldots, B_{b}\right\}$ be an ordered collection of $b$ blocks; each $B_{i}$ contains $k$ distinct elements from $[v]$.

We say that $\mathcal{B}$ has the single-change circular property if $\left|B_{i-1} \cap B_{i}\right|=k-1$ for $i=2, \ldots, b$, and $\left|B_{b} \cap B_{1}\right|=k-1$.

Lemma 2.1. $\quad \mathcal{B}$ is a $\operatorname{scccd}(v, k)$ if and only if

(i) $\mathcal{B}$ has the single-change circular property, and

(ii) every pair of $[v]$ is in some block of $\mathcal{B}$, and

(iii) no pair of $[v]$ is in every block of $\mathcal{B}$.

Proof. First suppose that $\mathcal{B}$ is a $\operatorname{scccd}(v, k)$. Then (i) and (ii) are true by definition of a scccd. Now suppose that (iii) is false, and that the pair $\{x, y\}$ is in every block of $\mathcal{B}$. Then neither $x$ nor $y$ is introduced in any block, hence $\{x, y\}$ is not covered by $\mathcal{B}$, a contradiction because $\mathcal{B}$ covers every pair.

Now suppose that $\mathcal{B}$ satisfies (i), (ii), and (iii). As $\mathcal{B}$ satisfies (i) we need only show that it covers every pair of $[v]$ to conclude that it is $\operatorname{a} \operatorname{scccd}(v, k)$. Now, by (ii), every pair $\{x, y\}$ lies in some block of $\mathcal{B}$ and, by (iii), the pair $\{x, y\}$ is not in every block. So let $B_{i_{1}}, B_{i_{2}}, \ldots, B_{i_{t}}$, where $t \leq b-1$, be a sequence of consecutive blocks each containing $\{x, y\}$; the block immediately 'before' $B_{i_{1}}$, say $B$, does not contain $\{x, y\}$. So exactly one of $x$ or $y$ lies in $B$ and the other does not. Hence, either $y$ or $x$ (respectively) is introduced in $B_{i_{1}}$, and so $\{x, y\}$ is covered there. Hence $\mathcal{B}$ covers every pair and is a $\operatorname{scccd}(v, k)$.

\section{Designs with $\mathrm{k}=2$ and 3}

$\mathbf{k}=\mathbf{2}$ If a tight $\operatorname{scccd}(v, 2, b)$ exists, then $b=v(v-1) / 2$, see $\S 5$. Now, given a tight $\operatorname{scccd}(v, 2)$ with first block $B_{1}=(1,2)$ and last block $B_{b}=(v, 1)$, we may add on the $v$ blocks as shown in Fig. 2(a) to obtain a tight $\operatorname{scccd}(v+1,2, v(v+1) / 2)$ based on $[v+1]$ with last block $(v+1,1)$.

Beginning with the tight $\operatorname{scccd}(3,2,3)$ shown in Fig. 2(b) we can use this construction repeatedly to obtain a tight $\operatorname{scccd}(v, 2)$ for any $v \geq 3$.

$\mathbf{k}=3$ If a tight $\operatorname{scccd}(v, 3, b)$ exists, then $b=v(v-1) / 4$, see $\S 5$; and so $v \equiv 0$ or $1(\bmod 4)$. Let $v \equiv 0(\bmod 4)$, and suppose that we have a tight $\operatorname{scccd}(v, 3)$ with $B_{1}=(1,2,3)$ and 
$B_{b}=(v, 1,2)$ in which element 1 is introduced in $B_{b}$. We can then construct a tight $\operatorname{scccd}(v+4,3,(v+3)(v+4) / 4)$ based on $[v+4]$ by altering $B_{b}$ to $(v, v+1,2)$ and adding on the $2 v+3$ blocks as shown in Fig. 2(c). This new design has last block $(v+4,1,2)$ in which 1 is introduced; so we can use this construction repeatedly to obtain a tight $\operatorname{scccd}(v, 3)$ for any $v \equiv 0(\bmod 4)$ beginning with the tight $\operatorname{scccd}(4,3,3)$ shown in Fig. 2(d).

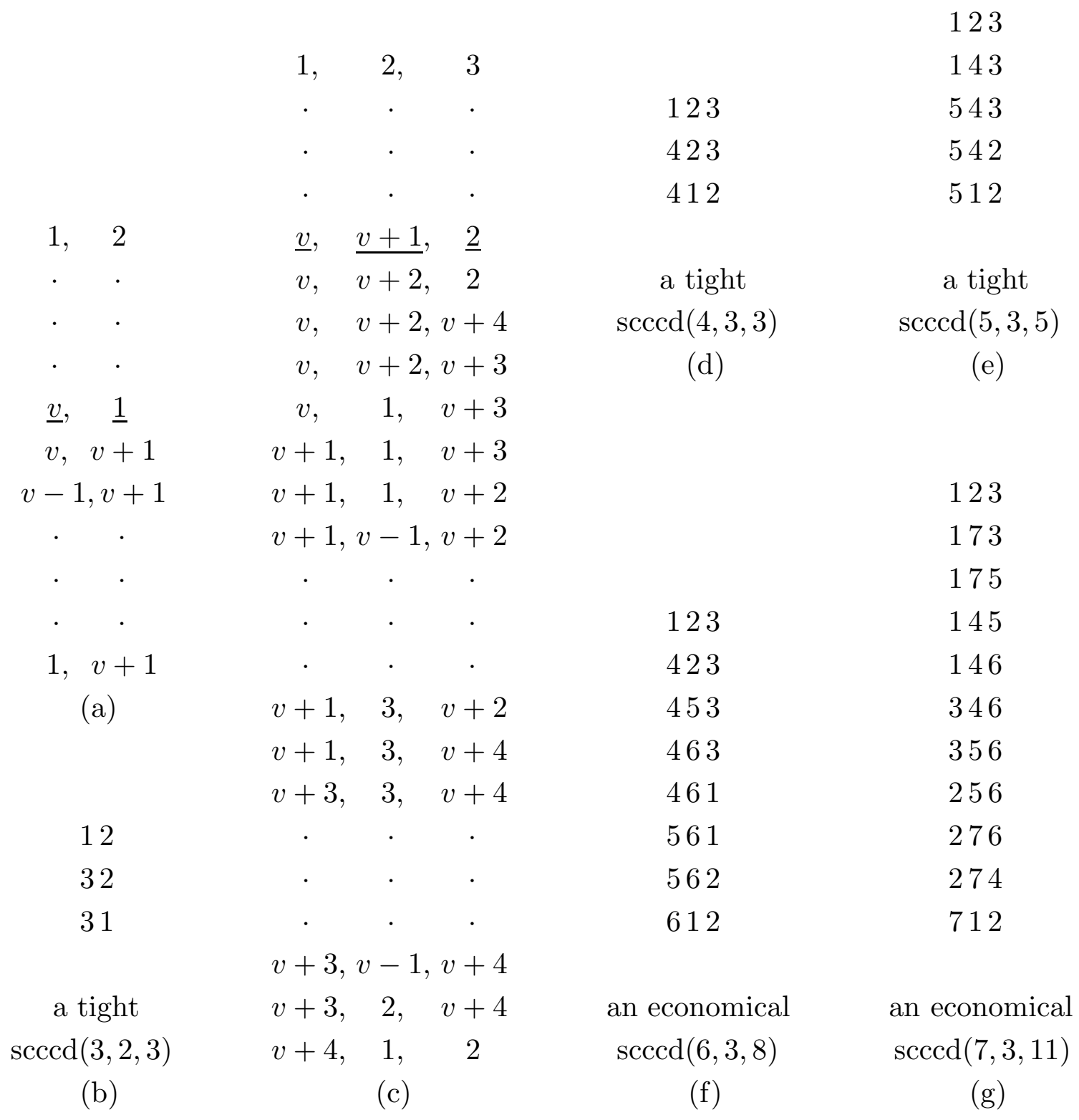

Fig. 2: Starter designs and additions for $k=2$ and 3. 
We can also construct a tight $\operatorname{scccd}(v, 3)$ when $v \equiv 1(\bmod 4)$ for any $v \geq 5$ starting with the tight $\operatorname{scccd}(5,3,5)$ shown in Fig. $2(\mathrm{e})$; and an economical $\operatorname{scccd}(v, 3,\lceil v(v-1) / 4\rceil)$ for $v \equiv 2$ or $3(\bmod 4)$ for any $v \geq 6$ starting with the economical scccds in (f) or $(\mathrm{g})$ respectively.

\section{Theorem 3.1.}

(i) A tight $\operatorname{scccd}(v, 2)$ exists for all $v \geq 3$;

(ii) a tight $\operatorname{scccd}(v, 3)$ exists for all $v \equiv 0$ or $1(\bmod 4), v \geq 4$;

(iii) an economical $\operatorname{scccd}(v, 3)$ exists for all $v \equiv 2$ or $3(\bmod 4), v \geq 6$.

\section{Standardized forms; isomorphisms; reverses}

$A \operatorname{scccd}(v, k, b)$ is standardized or in standardized form (see $\S 1$ of [2]]) if:

(1) the elements of the first block are $1,2, \ldots, k$ in that order;

(2) the other elements are introduced initially in the order $k+1, k+2, \ldots, v$;

(3) the elements of the first block are changed initially in the order $k, k-1, \ldots, 2,1$ (if our $\operatorname{scccd}(v, k, b)$ has one element, say element 1 , in every block, then the elements of the first block are changed initially in the order $k, k-1, \ldots, 2)$;

(4) beginning at the second block, a block's unchanged elements are in the same columns as in the previous block (i.e., it is column-strict).

Given any $\operatorname{scccd}(v, k, b), \mathcal{B}$, that satisfies (4) above, in order to change it to its standardized form we need to apply a permutation of $[v]$ to it, followed by a permutation of its columns. For example, if we apply the permutation $(1,6)(3,4)$ to $\mathcal{E}=\left\{B_{1}, B_{2}, \ldots, B_{8}\right\}$ shown in Fig. 3(a), and then permute its 2nd and 3rd columns, we arrive at its standardized form shown in (b), with blocks labelled $L_{i}$.

A cyclic shift of the ordered blocks $\mathcal{B}=\left\{B_{1}, B_{2}, \ldots, B_{b}\right\}$ is one of the following rearrangements:

$$
\begin{array}{rcc}
\mathcal{B}=\mathcal{B}_{1}= & \left\{B_{1}, B_{2}, \ldots, B_{b}\right\} \\
\mathcal{B}_{2}= & \left\{B_{2}, B_{3}, \ldots, B_{b}, B_{1}\right\}, \\
\cdot & \cdot & \cdot \\
\cdot & \cdot & \cdot \\
\mathcal{B}_{i}= & \left\{B_{i}, \ldots, B_{b}, B_{1}, \ldots, B_{i-1}\right\} \\
\cdot & \cdot & \cdot \\
\cdot & \cdot & \cdot \\
\mathcal{B}_{b}= & \left\{B_{b}, B_{1}, \ldots, B_{b-1}\right\} .
\end{array}
$$

The block arrangement $\mathcal{B}_{i}$ is called the $i$-th cyclic shift of $\mathcal{B}$. For each $i$ the first block in $\mathcal{B}_{i}$ is $B_{i}$. 


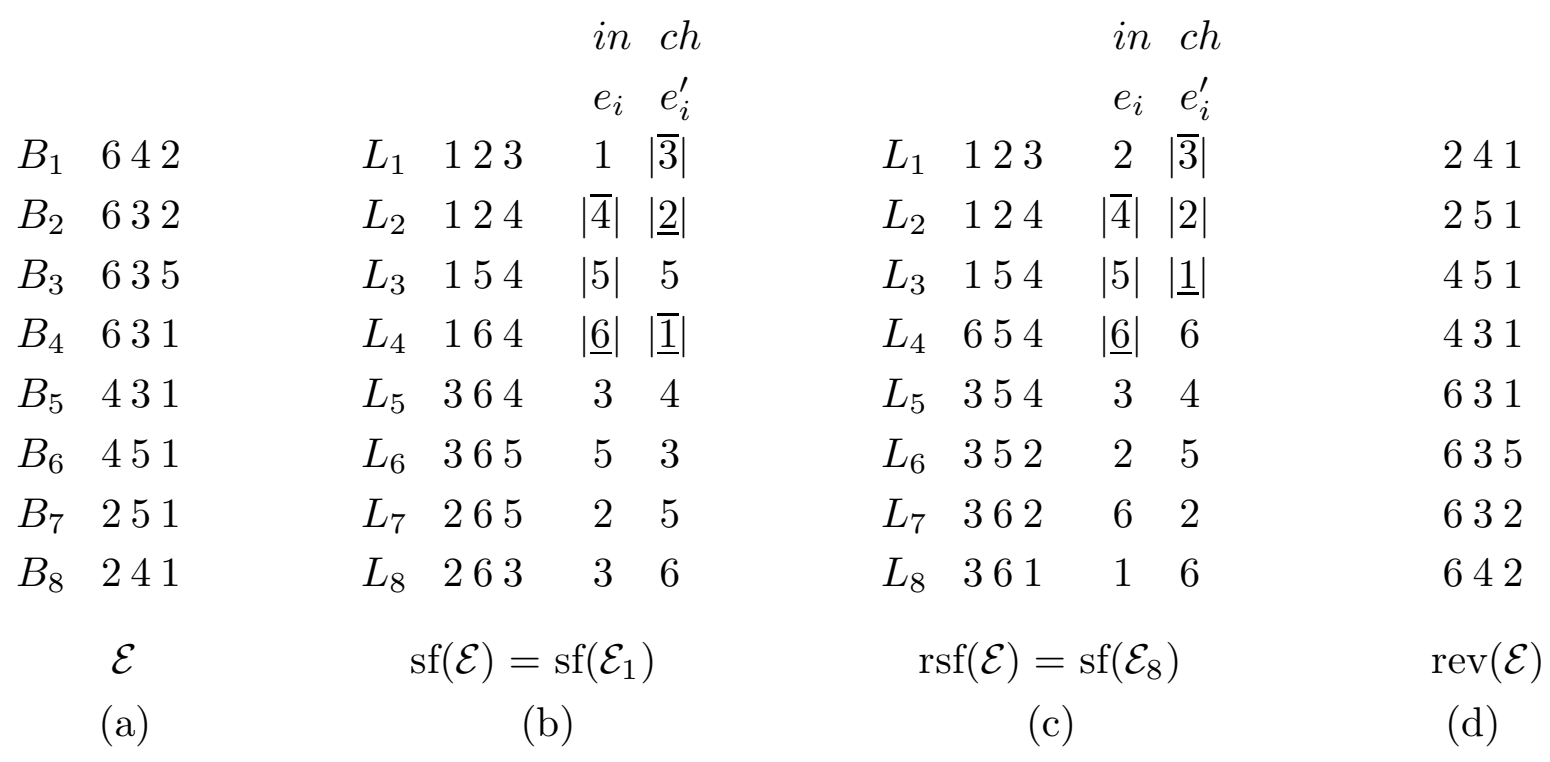

Fig. 3: The economical $\operatorname{scccd}(6,3,8), \mathcal{E}$, its standardized form, its representative standardized form, and its reverse.

Each scced $\mathcal{B}=\left\{B_{1}, B_{2}, \ldots, B_{b}\right\}$ has $b$ standardized forms, one for each cyclic shift $\mathcal{B}_{i}$; let $\operatorname{sf}\left(\mathcal{B}_{i}\right)$ denote the standardized form of $\mathcal{B}_{i}$ for each $i=1, \ldots, b$.

The $\operatorname{scccd}(5,3,5), \mathcal{Y}_{3}$, of Fig. 1(b) has each of its 5 standardized forms identical (shown in Fig. 15(d)); but $\mathcal{E}$ in Fig. 3(a) has each of its 8 standardized forms different. If a design $\mathcal{B}$ does not have all of its standardized forms identical, one of these forms can usefully be chosen as the representative standardized form $\operatorname{rsf}(\mathcal{B})$. In order to do this for an arbitrary $\mathcal{B}=\left\{B_{1}, B_{2}, \ldots, B_{b}\right\}$, we will presently define, for each $i$, four finite sequences associated with $\operatorname{sf}\left(\mathcal{B}_{i}\right)$, namely $S_{1}, S_{2}, S_{3}$, and $S_{4}$. (In general, the sequences $S_{1}, S_{2}, S_{3}$, and $S_{4}$ will be different for each $i$, but we choose this notation for simplicity.) We then consider the ordered set $\left\{S_{1}, S_{2}, S_{3}, S_{4}\right\}$. So each $\operatorname{sf}\left(\mathcal{B}_{i}\right)$ gives us an ordered set of four sequences. Next, we order these ordered sets of four sequences according to the criteria below, and choose the 'least' in this ordering. Suppose this least ordered set comes from $\operatorname{sf}\left(\mathcal{B}_{\ell}\right)$, then $\operatorname{sf}\left(\mathcal{B}_{\ell}\right)$ is taken as $\operatorname{rsf}(\mathcal{B})$.

Again for simplicity, we use $L_{1}, L_{2}, \ldots, L_{b}$, to denote the $b$ blocks of $\operatorname{sf}\left(\mathcal{B}_{i}\right)$, for every $i=1, \ldots, b$, even though generally the blocks differ for each $i$. $\operatorname{Sosf}\left(\mathcal{B}_{i}\right)=\left\{L_{1}, L_{2}, \ldots, L_{b}\right\}$ where $L_{1}=(1,2, \ldots, k)$ and $L_{2}=(1,2, \ldots, k+1)$.

The sequences $S_{1}$ and $S_{2}$ are sequences of distinct blocks from $\left\{L_{1}, L_{2}, \ldots, L_{b}\right\}$; and $S_{3}$ and $S_{4}$ are sequences of not necessarily distinct elements from $[v]$. For a fixed $\operatorname{sf}\left(\mathcal{B}_{i}\right)$ we define $S_{1}$ with reference to (2) above. For $t=1, \ldots, v-k$, the $t$-th member of $S_{1}$ is the block into which element $k+t$ is initially introduced. Thus $S_{1}$ begins with $L_{2}$. Sequence $S_{2}$ (see (3) above) is a sequence of blocks beginning at $L_{1}$. For $t=1, \ldots, k$, the $t$-th member 
of $S_{2}$ is the first block from which element $k-t+1$ is initially changed ( $t$ stops at $k-1$ if $\mathcal{B}$ has element 1 in every block).

Just as for $i=1, \ldots, b$, the element $e_{i}$ is introduced into block $L_{i}$, let $e_{i}^{\prime}$ be the element changed from $L_{i}$. So we have $e_{i}^{\prime} \in L_{i} \backslash L_{i+1}$ for $i=1, \ldots, b-1$, and $e_{b}^{\prime} \in L_{b} \backslash L_{1}$. Now define sequence $S_{3}=\left\{e_{i}: L_{i} \notin S_{1}\right\}$, arranged with increasing $i$; so, $e_{1}$ is its first member. Similarly, we define $S_{4}=\left\{e_{i}^{\prime}: L_{i} \notin S_{2}\right\}$, arranged with increasing $i$.

For an example see $\operatorname{sf}\left(\mathcal{E}_{1}\right)$ in Fig. 3(b), where the column of introduced elements, $e_{i}$, is labelled 'in', and the column of changed elements, $e_{i}^{\prime}$, is labelled 'ch'. We have $S_{1}=\left\{L_{2}, L_{3}, L_{4}\right\}, S_{2}=\left\{L_{1}, L_{2}, L_{4}\right\}, S_{3}=\{1,3,5,2,3\}$, and $S_{4}=\{5,4,3,5,6\}$.

Thus each $\operatorname{sf}\left(\mathcal{B}_{i}\right)$ gives us an ordered set of four sequences. We now order these ordered sets of four sequences by, first of all, lexicographically ordering their first elements, the $S_{1}$ sequences, according to the rule: $L_{r}<L_{s}$ if and only if $r<s$, and choosing the set(s) whose $S_{1}$ sequence is the first (i.e., the least) in this list. If two (or more) sets have identical $S_{1}$ sequences, we choose the one with the least $S_{2}$ sequence, using the same ordering. If two (or more) sets have identical $S_{1}$ and $S_{2}$ sequences we then compare their $S_{3}$ sequences and order them lexicographically using the natural < ordering on $[v]$, and choose the least. If still identical, we compare their $S_{4}$ sequences, with the < ordering, and choose the least. Two such sets with identical $S_{1}, S_{2}, S_{3}$, and $S_{4}$ sequences can easily be shown to correspond to standardized forms that are identical.

By this process we arrive at the particular $\operatorname{sf}\left(\mathcal{B}_{\ell}\right)$ with the least set of sequences according to our lexicographic orderings; we take this particular standardized form as the representative standardized form of $\mathcal{B}, \operatorname{rsf}(\mathcal{B})$. For example, $\operatorname{rsf}(\mathcal{E})$, shown in Fig. 3(c), is $\operatorname{sf}\left(\mathcal{E}_{8}\right)$; and $\operatorname{rsf}\left(\mathcal{Y}_{3}\right)$ is shown in Fig. 15(d).

Two $\operatorname{scccd}(v, k, b) \mathrm{s} \mathcal{B}$ and $\mathcal{B}^{\prime}$ are isomorphic, $\left(\mathcal{B} \cong \mathcal{B}^{\prime}\right)$, if we can apply a permutation of $[v]$ combined with a cyclic shift of the blocks of $\mathcal{B}$ to obtain $\mathcal{B}^{\prime}$. Similarly, an automorphism of a $\operatorname{scccd}(v, k, b) \mathcal{B}$ is a permutation of $[v]$ which, when applied to $\mathcal{B}$, produces a cyclic shift of $\mathcal{B}$. For example, the permutation $(1,2,3,4,5)$ is an automorphism of $\mathcal{Y}_{3}$.

Theorem 4.1. Let $\mathcal{B}$ and $\mathcal{B}^{\prime}$ be two $\operatorname{scccd}(v, k, b) \mathrm{s}$.

Then $\mathcal{B} \cong \mathcal{B}^{\prime}$ if and only if $\operatorname{rsf}(\mathcal{B})=\operatorname{rsf}\left(\mathcal{B}^{\prime}\right)$.

Proof. Suppose $\mathcal{B} \cong \mathcal{B}^{\prime}$, then, for any $i \in\{1, \ldots, b\}$, there exists a $j \in\{1, \ldots, b\}$ such that $\mathcal{B}_{i}$, the $i$-th cyclic shift of $\mathcal{B}$, can be changed into $\mathcal{B}_{j}^{\prime}$ using only a permutation on $v$, i.e., with no cyclic shift of the blocks of $\mathcal{B}_{i}$. $\operatorname{So~} \operatorname{sf}\left(\mathcal{B}_{i}\right)=\operatorname{sf}\left(\mathcal{B}_{j}^{\prime}\right)$, and so $\left\{\operatorname{sf}\left(\mathcal{B}_{1}\right), \ldots, \operatorname{sf}\left(\mathcal{B}_{b}\right)\right\} \subseteq$ $\left\{\operatorname{sf}\left(\mathcal{B}_{1}^{\prime}\right), \ldots, \operatorname{sf}\left(\mathcal{B}_{b}^{\prime}\right)\right\}$. Similarly, $\left\{\operatorname{sf}\left(\mathcal{B}_{1}^{\prime}\right), \ldots, \operatorname{sf}\left(\mathcal{B}_{b}^{\prime}\right)\right\} \subseteq\left\{\operatorname{sf}\left(\mathcal{B}_{1}\right), \ldots, \operatorname{sf}\left(\mathcal{B}_{b}\right)\right\}$. Finally we see that $\left\{\operatorname{sf}\left(\mathcal{B}_{1}\right), \ldots, \operatorname{sf}\left(\mathcal{B}_{b}\right)\right\}=\left\{\operatorname{sf}\left(\mathcal{B}_{1}^{\prime}\right), \ldots, \operatorname{sf}\left(\mathcal{B}_{b}^{\prime}\right)\right\}$, and so $\operatorname{rsf}(\mathcal{B})=\operatorname{rsf}\left(\mathcal{B}^{\prime}\right)$.

Now suppose that $\operatorname{rsf}(\mathcal{B})=\operatorname{rsf}\left(\mathcal{B}^{\prime}\right)$, then $\mathcal{B} \cong \operatorname{rsf}(\mathcal{B})=\operatorname{rsf}\left(\mathcal{B}^{\prime}\right) \cong \mathcal{B}^{\prime}$, as required. 
The reverse of the scccd $\mathcal{B}=\left\{B_{1}, B_{2}, \ldots, B_{b}\right\}$ is the scccd obtained by reversing the order of the blocks of $\mathcal{B}$; we denote this design by $\operatorname{rev}(\mathcal{B})=\left\{B_{b}, B_{b-1}, \ldots, B_{1}\right\}$. The reverse of $\mathcal{E}$ is shown in Fig. $3(\mathrm{~d})$. If $\mathcal{B} \cong \operatorname{rev}(\mathcal{B})$ we say that $\mathcal{B}$ is self-reverse.

\section{Lower bounds on $b_{*}(v, k)$; constructions of designs for $k+1 \leq v \leq 2 k$}

Lemma 5.1. For $v \geq 4$ and $k \geq 3$, the value of $b_{*}(v, k)$, the minimum number of blocks in $\operatorname{acccd}(v, k)$, satisfies

$$
b_{*}(v, k) \geq \max \left\{v-1,\left\lceil\frac{v(v-1)}{2(k-1)}\right\rceil\right\} .
$$

Proof. In a $\operatorname{scccd}(v, k)$ exactly one element is introduced per block, so, if $b_{*}(v, k)<v-1$, then at most $v-2$ distinct elements are introduced. Hence, at least 2 distinct elements are not introduced and the pair containing them is not covered, a contradiction. So $b_{*}(v, k) \geq v-1$.

$\mathrm{A} \operatorname{scccd}(v, k)$ must cover all $v(v-1) / 2$ pairs of $[v]$, and $k-1$ pairs are covered per block. Thus $b_{*}(v, k) \cdot(k-1) \geq v(v-1) / 2$, and so the result.

Corollary 5.2. For $v \geq 4$ and $k \geq 3$ we have

$$
b_{*}(v, k) \geq \begin{cases}v-1, & \text { for } k+1 \leq v \leq 2 k-2 \\ \left\lceil\frac{v(v-1)}{2(k-1)}\right\rceil, & \text { for } v>2 k-2\end{cases}
$$

If $b_{*}(v, k)$ satisfies equation (1) with equality, then the corresponding $\operatorname{scccd}(v, k)$ is economical; except that if $b_{*}(v, k)=\frac{v(v-1)}{2(k-1)}$ then the corresponding $\operatorname{scccd}(v, k)$ is tight. An economical and a tight design are shown in Fig. 1.

Consider the first case of Corollary 5.2. If a $\operatorname{scccd}(v, k)$ with $k+1 \leq v \leq 2 k-2$ and with the minimal number of $v-1$ blocks exists, then a total of $v-1$ elements are introduced in the design; from the proof of Lemma 5.1 these $v-1$ elements are distinct. Hence, without loss of generality, in such a design the elements $1, \ldots, v-1$ are each introduced exactly once; the element $v$ is not introduced, and so appears in every block. The following construction satisfies these requirements:

Arrange the elements of $[v-1]$ in a circle and call this arrangement the circular $[v-1]$. Now, for $1 \leq i \leq v-1$, let $A_{i}$ be the block beginning at $i$ and containing $k-1$ consecutive elements taken clockwise from the circle; i.e., $A_{i}=(i, i+1, \ldots, i+k-2)$, where addition is taken modulo $v-1$ with $v-1$ replacing 0 . Now let $B_{i}=A_{i} \cup\{v\}$ and $\mathcal{B}=\left\{B_{1}, \ldots, B_{v-1}\right\}$. 
Theorem 5.3. For $k \geq 3$ and $k+1 \leq v \leq 2 k-2$ the blocks $\mathcal{B}=\left\{B_{1}, \ldots, B_{v-1}\right\}$ where $B_{i}=A_{i} \cup\{v\}$ form an economical $\operatorname{scccd}(v, k, v-1)$.

Proof. We need only show that $\mathcal{B}$ is a $\operatorname{scccd}(v, k)$. It then follows, since $|\mathcal{B}|=v-1$, that it is an economical $\operatorname{scccd}(v, k, v-1)$. We show that $\mathcal{B}$ satisfies (i), (ii), and (iii) of Lemma 2.1 .

(i) This is clear from the definition of $\mathcal{B}$.

(ii) Now $B_{i}=(i, i+1, \ldots, i+k-2) \cup\{v\}$, where addition is taken modulo $v-1$ with $v-1$ replacing 0 . So element $i+k-2$ is introduced in $B_{i}$, i.e., element $i$ is introduced in $B_{i-k+2}$. Hence, for $1 \leq i \leq v-1$, pair $\{i, v\}$ is covered by $B_{i-k+2}$. This deals with pairs that contain $v$.

Now consider the pair $\{i, j\}$ where $1 \leq i<j \leq v-1$ and let $v$ be even. The pair $\{i, j\}$ can be covered by a 'run' of $v / 2$ consecutive elements of the circular $[v-1]$ starting either at $i$ or at $j$. But $v \leq 2 k-2$ and so $v / 2 \leq k-1$, i.e., such a run is contained in $A_{i}$ or $A_{j}$, so in $B_{i}$ or $B_{j}$. Hence $\{i, j\}$ is covered by $\mathcal{B}$. A similar argument works when $v$ is odd.

(iii) Let $\{i, j\}$ be in every block; then, without loss of generality, we have $1 \leq i \leq v-1$. But $i$ is introduced in $B_{i-k+2}$, and so cannot be in the previous block, a contradiction. Hence no pair is in every block.

Thus $\mathcal{B}$ is an economical $\operatorname{scccd}(v, k, v-1)$.

An economical scccd $(5,4,4)$ constructed using Theorem 5.3 is shown in Fig. 4(a).

$\begin{array}{ccc} & & B_{1} 1234 \\ & & B_{2} 2345 \\ & B_{1} 1236 & B_{3} 3456 \\ B_{1} 1235 & B_{2} 2346 & B_{4} 4567 \\ B_{2} 2345 & B_{3} 3456 & B_{5} 5671 \\ B_{3} 3415 & B_{4} 4516 & B_{6} 6712 \\ B_{4} 4125 & B_{5} 5126 & B_{7} 7123 \\ & & \\ \text { an economical } & \text { a tight } & \text { a tight } \\ \operatorname{scccd}(5,4,4) & \text { scccd(6, }(6,5) & \text { scccd }(7,4,7) \\ \text { (a) } & \text { (b) } & \text { (c) }\end{array}$

Fig. 4: Designs with $k=4$ constructed using Theorems 5.3, 5.4, and 5.5 respectively. 
For a fixed $k \geq 3$ the only tight designs amongst the economical $\operatorname{scccd}(v, k, v-1) \mathrm{s}$ with $k+1 \leq v \leq 2 k-2$ occur when $v(v-1) / 2=(k-1)(v-1)$, i.e., when $v=2 k-2$. So Theorem 5.3 with $v=2 k-2$ yields tight designs:

Theorem 5.4. For $k \geq 3$ and $v=2 k-2$ the blocks $\mathcal{B}=\left\{B_{1}, \ldots, B_{2 k-3}\right\}$ where $B_{i}=A_{i} \cup\{2 k-2\}$ form a tight $\operatorname{scccd}(2 k-2, k, 2 k-3)$.

See Fig. 4(b) for a tight $\operatorname{scccd}(6,4,5)$ constructed using Theorem 5.4.

So far, for a fixed $k \geq 3$, we have constructed economical scccds when $k+1 \leq v \leq$ $2 k-2$. We now consider $v=2 k-1$ and $v=2 k$, so we are in the second case of Corollary 5.2 in which a scccd has $b \geq\left\lceil\frac{v(v-1)}{2(k-1)}\right\rceil$ blocks.

$\mathbf{v}=\mathbf{2 k - 1}$ Here $b \geq 2 k-1$. Consider the circular $[2 k-1]$. For $1 \leq i \leq 2 k-1$ let $B_{i}=(i, i+1, \ldots, i+k-1)$, where addition is taken modulo $2 k-1$ with $2 k-1$ replacing 0 . Then we have the following result. This construction and the next also work for $k=2$.

Theorem 5.5. For $k \geq 2$ and $v=2 k-1$ the blocks $\mathcal{B}=\left\{B_{1}, \ldots, B_{2 k-1}\right\}$ where $B_{i}=(i, i+1, \ldots, i+k-1)$ form a tight $\operatorname{scccd}(2 k-1, k, 2 k-1)$.

See Fig. 4(c) for a tight $\operatorname{scccd}(7,4,7)$ constructed using Theorem 5.5.

$\mathbf{v}=\mathbf{2 k}$ Here $b \geq 2 k+2$. To construct an economical $\operatorname{scccd}(2 k, k, 2 k+2)$ based on $[2 k]$ we take the blocks $\left\{B_{1}, \ldots, B_{2 k-1}\right\}$ of the $\operatorname{scccd}(2 k-1, k, 2 k-1)$ in Theorem 5.5 above and add on 3 new blocks: $C$ between $B_{k-1}$ and $B_{k}$, then $C^{\prime}$ after $B_{2 k-1}$, and finally $C^{\prime \prime}$ after $C^{\prime}$, i.e., between $C^{\prime}$ and $B_{1}$; see Fig. $5($ a).

In Fig. 5(a) the single-change circular property between the blocks is preserved. Further, as the elements introduced in $B_{1}$ and $B_{k}$ are unchanged, the pairs covered by these blocks are unchanged; and pairs containing the new element $2 k$ are covered in the 3 new blocks, thus all pairs are covered. Finally, no pair is in every block. Thus, from Lemma 2.1, this is a $\operatorname{scccd}(2 k, k, 2 k+2)$, which is economical; it is tight only when $2 k+2=\frac{2 k(2 k-1)}{2(k-1)}$, i.e., when $k=2$.

\section{Theorem 5.6.}

For $k \geq 2$ and $v=2 k$ the blocks $\mathcal{B}=\left\{B_{1}, B_{2}, \ldots, B_{k-1}, C, B_{k}, \ldots, B_{2 k-1}, C^{\prime}, C^{\prime \prime}\right\}$ form an economical $\operatorname{scccd}(2 k, k, 2 k+2)$, which is tight when $k=2$.

See Fig. 5(b) for an economical scccd $(8,4,10)$ constructed using Theorem 5.6. 


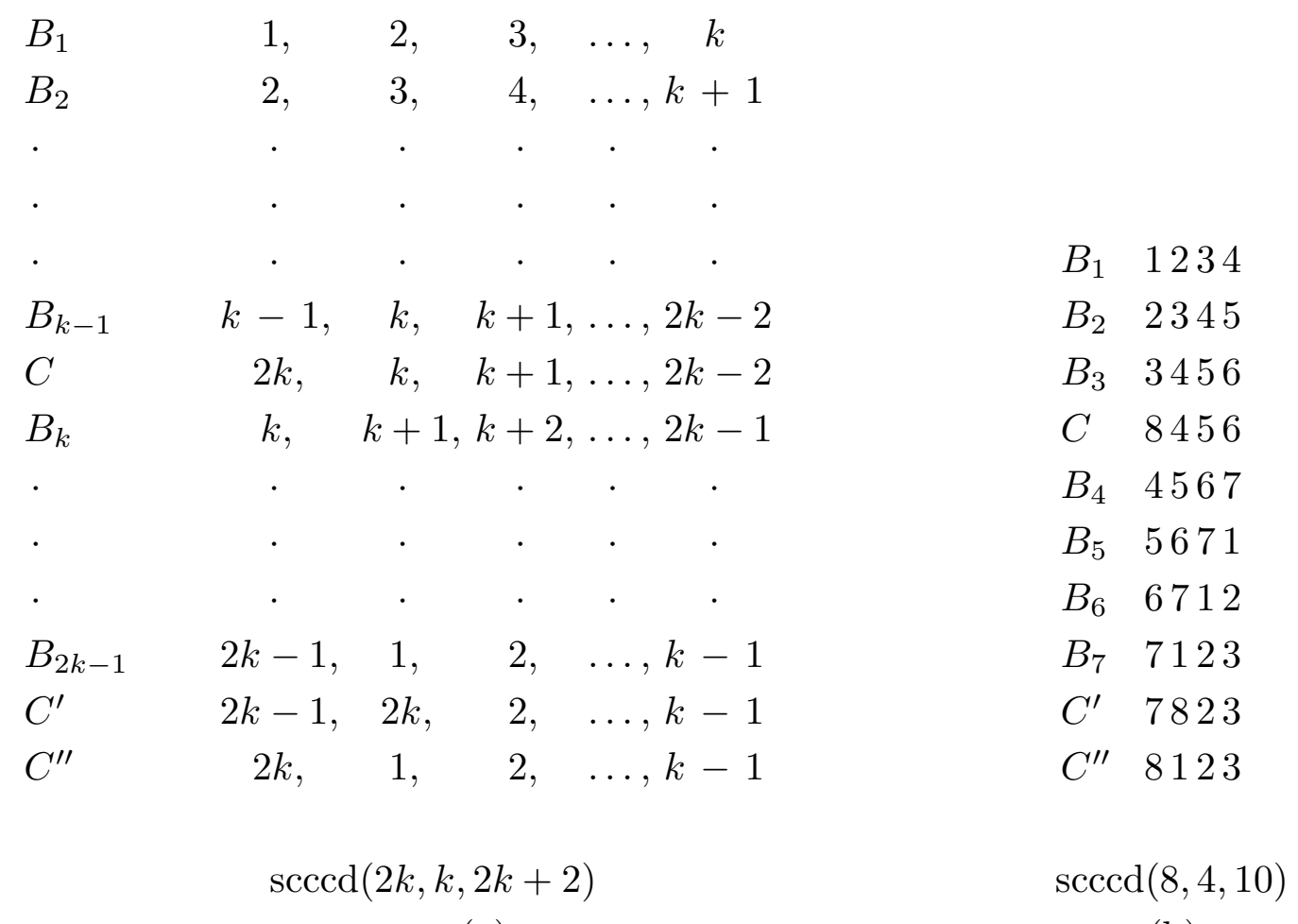

(a)

Fig. 5: The economical $\operatorname{scccd}(2 k, k, 2 k+2)$ of Theorem 5.6 (tight only for $k=2$ ), this design for $k=4$.

The theorem below summarizes this section, cf., Corollary 5.1 of [ [ $]$.

Theorem 5.7. $\quad$ For $k \geq 3$ we have

$$
b_{*}(v, k)= \begin{cases}v-1, & \text { for } k+1 \leq v \leq 2 k-2 \\ v, & \text { for } v=2 k-1 \\ v+2, & \text { for } v=2 k\end{cases}
$$

So, in this section, we have constructed scccds with a minimal number of blocks for all $k \geq 3$ and $k+1 \leq v \leq 2 k$, and have given examples for $k=4$.

From now on we concentrate mainly on tight designs. 


\section{Some families of tight designs}

Tight scccds are of special interest; they are analogous to tight single-change covering designs, see $[\underline{2}]$.

So far we have three infinite families of tight scccds with a fixed $k$, see Theorem 3.1:

(i) $\operatorname{scccd}(v, 2)$ for all $v \geq 3$,

(ii) $\operatorname{scccd}(v, 3)$ for all $v \equiv 0(\bmod 4), v \geq 4$, and

(iii) $\operatorname{sccd}(v, 3)$ for all $v \equiv 1(\bmod 4), v \geq 5$.

For the tight designs of [2] infinite families are not known to exist with $k$ variable, except in the case $v=k$, here we have two infinite families with $k$ variable:

(iv) $\mathfrak{F}=\left\{\mathcal{X}_{k}: \mathcal{X}_{k}\right.$ is the tight $\operatorname{scccd}(2 k-2, k, 2 k-3)$ of Theorem $\left.5.4, k \geq 3\right\}$;

(v) $\mathfrak{G}=\left\{\mathcal{Y}_{k}: \mathcal{Y}_{k}\right.$ is the tight $\operatorname{scccd}(2 k-1, k, 2 k-1)$ of Theorem $\left.5.5, k \geq 2\right\}$. (Note that $\mathcal{Y}_{3}$ is shown in Fig. 1(b).)

If a scced has the same parameters as a member of $\mathfrak{F}$ or $\mathfrak{G}$ then it is isomorphic to that member:

Theorem 6.1. For a fixed $k \geq 3$ let $\mathcal{X}$ be a $\operatorname{scccd}(2 k-2, k, 2 k-3)$.

Then $\mathcal{X} \cong \mathcal{X}_{k} \in \mathfrak{F}$.

Proof. The parameters of $\mathcal{X}$ indicate that it is tight. Let $\mathcal{X}$ be based on $[v]$ where $v=2 k-2$.

Now, $\mathcal{X}$ has $v-1$ blocks, so, from the comments preceding Theorem 5.3, it contains some element, say $v$, in every block. Any other element $1, \ldots, v-1$ is introduced exactly once and remains in $k-1$ successive blocks because it must appear in $v-1=2 k-3$ covered pairs; it appears in $k-1$ covered pairs in its first block and in one covered pair in each of its $k-2$ successive blocks.

Hence, up to a permutation of $[v]$, we may construct $\mathcal{X}$ as follows: first, put $v$ in every block; then, for $i=1, \ldots, v-1$, introduce $i$ in $B_{i}$, and leave it there for $k-1$ successive blocks. Then $B_{k-1}=(1,2, \ldots, k-1, v)$, and a cyclic shift of the blocks making this the first block will produce $\mathcal{X}_{k}$. Hence $\mathcal{X} \cong \mathcal{X}_{k}$.

Similarly for the family $\mathfrak{G}$.

Theorem 6.2. For a fixed $k \geq 2$ let $\mathcal{Y}$ be a $\operatorname{scccd}(2 k-1, k, 2 k-1)$. Then $\mathcal{Y} \cong \mathcal{Y}_{k} \in \mathfrak{G}$. 


\section{The numbers $t_{j}$ and $f_{j}$ for a tight design}

Now we consider constructions of tight scccds for $v>2 k$. First we need some preparatory material, much of which is similar to that of $\S 4$ in $[2]$.

In an arbitrary tight $\operatorname{scccd}(v, k)$ let $T_{j} \subseteq[v]$ denote the set of elements which are introduced $j$ times, $j \geq 0$, and let $t_{j}=\left|T_{j}\right|$.

Now consider $t_{0}$, the number of elements not introduced. From the proof of Lemma 5.1 we must have $t_{0}=0$ or 1 . Let $\mathcal{Z}$ be a tight $\operatorname{scccd}(v, k)$ in which $t_{0}=1$, and let $v$ be the element not introduced, so $v$ is in every block. Any other element $z=1, \ldots, v-1$ is introduced exactly once, for, if some $z$ is introduced twice or more, then the pair $\{z, v\}$ is covered twice or more; a contradiction because $\mathcal{Z}$ is tight. Hence, each of $1, \ldots, v-1$ is introduced exactly once, and $b=v-1=\frac{v(v-1)}{2(k-1)}$, i.e., $v=2 k-2$. Thus $\mathcal{Z}$ is a tight $\operatorname{scccd}(2 k-2, k, 2 k-3)$ and, by Theorem 6.1 , lies in $\mathfrak{F}$.

Thus, all tight scccds with $t_{0}=1$ are known; they are members of $\mathfrak{F}$ with $v=2 k-2$. As we are interested in tight designs with $v>2 k$, we assume that $t_{0}=0$ and consider only $t_{j}$ for which $j \geq 1$.

Let $\mathcal{C}$ be an arbitrary tight $\operatorname{scccd}(v, k, b)$, and, for any $x \in[v]$, let $f_{\{x\}}$ denote the number of blocks that contain $x$.

Let $x \in T_{j}$. Each time $x$ is introduced $k-1$ pairs containing $x$ are covered. There are $v-1$ pairs containing $x$ to be covered, so we may let $j \leq\left\lfloor\frac{v-1}{k-1}\right\rfloor=A$ because $t_{j}=0$ for $j>A$.

Now $x \in T_{j}$, so there are $j$ blocks in which $x$ is introduced; $(k-1)$ pairs containing $x$ are covered in each of these blocks. There are $f_{\{x\}}-j$ blocks that contain $x$ but in which it is not introduced; only 1 pair containing $x$ is covered in each of these blocks. This gives $v-1=j(k-1)+\left(f_{\{x\}}-j\right) 1$, i.e., $f_{\{x\}}=(v-1)-j(k-2)$. Hence $f_{\{x\}}$ is constant on $T_{j}$, so we let $f_{j}=(v-1)-j(k-2)$ be the number of blocks that contain any fixed element from $T_{j}$. In particular, $f_{1}=v-k+1$.

We have, for $1 \leq j \leq A$,

$$
A=\left\lfloor\frac{v-1}{k-1}\right\rfloor, \quad \sum_{j=1}^{A} t_{j}=v, \quad \sum_{j=1}^{A} j t_{j}=b, \quad f_{j}=(v-1)-j(k-2) .
$$

Some further properties of the numbers $t_{j}$ and $f_{j}$ are given below.

Lemma 7.1. For $k>2$ and any $j$ for which $1 \leq j \leq A$, we have

(i) $f_{A}<f_{A-1}<\cdots<f_{2}<f_{1}$;

(ii) $f_{j} \geq j$, and, if $f_{j}<j$, then $t_{\ell}=0$ for all $\ell \geq j$;

(iii) if $f_{j}=j$, then $t_{j}=0$ or 1 ;

(iv) if $f_{j}=j+1$, then $t_{j}=0,1$, or 2 . 


\section{Proof.}

(i) Clear because $f_{j}=(v-1)-j(k-2)$ and $k>2$.

(ii) For each of the $j$ times when $x \in T_{j}$ is introduced it appears in at least 1 block, so $f_{j} \geq j$. So, clearly, if $f_{j}<j$ then $t_{j}=0$. Also, for any $\ell>j$, we have $f_{\ell}<f_{j}<j<\ell$ by (i), hence $t_{\ell}=0$.

(iii) For a fixed $j$, suppose $f_{j}=j$ but $t_{j}=\left|T_{j}\right| \geq 2$, and let $x$ and $y \in T_{j}$. Now, because $x$ is introduced $j$ times and appears in $j$ blocks, each time it is introduced it must be immediately removed; similarly for $y$. But pair $\{x, y\}$ must appear in some block, hence both $x$ and $y$ must be introduced in this block, a contradiction.

(iv) Now suppose $f_{j}=j+1$ but $t_{j} \geq 3$, and let $x, y$, and $z \in T_{j}$. By the pigeonhole principle for one of the $j$ times when $x$ is introduced it must stay for 2 successive blocks; similarly for $y$ and $z$. So the configurations $\underset{x}{x}, \underset{y}{y}$, and $\underset{z}{z}$ occur once each. In order to cover the pairs $\{x, y\},\{x, z\}$, and $\{y, z\}$ we must have the arrangement $\begin{aligned} & x z y \\ & z y\end{aligned}$. in the design, i.e., $b=3$. But there are only 2 tight scccds with $b=3$ : one is $\mathcal{X}_{3} \in \mathfrak{F}$, which we have excluded; the other is $\mathcal{Y}_{2} \in \mathfrak{G}$, which is also excluded because this design has $k=2$ and we are restricted to $k>2$.

\section{Start-Finish arrays for a tight design; Criteria for a tight design with $\mathrm{v}>2 \mathrm{k}$}

This section is mainly concerned with the subset $T_{1}$ of elements introduced exactly once in $\mathcal{C}$, an arbitrary tight $\operatorname{scccd}(v, k)$.

Suppose $T_{1} \neq \emptyset$ and let $x \in T_{1}$, and consider the $f_{\{x\}}=f_{1}=v-k+1$ successive blocks in $\mathcal{C}$ which contain $x$; call these blocks $\mathcal{B}_{x}=\left\{B_{x, 1}, \ldots, B_{x, f_{1}}\right\}$, see Fig. 6(a). We may write $x$ as the leftmost element in each of these blocks. We say that $x$ starts, $S$, in $B_{x, 1}$ (i.e., is introduced there), and finishes, $F$, in $B_{x, f_{1}}$, see Fig. 6(a). Call this occurrence of $x$ in $f_{1}$ successive blocks the run containing $x$. Now let $y$ be some other element in $T_{1}$. The pair $\{x, y\}$ must be covered in $\mathcal{B}_{x}$ and so, because $f_{\{y\}}=f_{1}$ also, either the $S$ or the $F$ of $y$ must appear in $\mathcal{B}_{x}$. Similarly for all the other elements in $T_{1}$.

Thus, as we run through the elements in $T_{1}$, each adds its $S$ or its $F$ to the array in the final column of Fig. 6(a). We call this the Start-Finish array, or $S F$-array, for element $x$, and denote it by $S F_{x}$. Call the $S$ s and $F$ s symbols.

In Fig. 6(a), block $B_{x, i}$ gives rise to the $i$-th row, $R_{x, i}$, of $S F_{x}$, which contains $x$ and, perhaps, some symbols; to illustrate this we write $B_{x, i} \rightarrow R_{x, i}$. If a row contains no symbols it is empty (-). 


\begin{tabular}{|c|c|c|c|c|c|c|c|c|c|c|}
\hline$B_{x, 1}$ & $\rightarrow R_{x, 1}$ & $x S$ & & & & & & & & \\
\hline$B_{x, 2}$ & $\rightarrow R_{x, 2}$ & $x$ & 12 & & & & & & & \\
\hline$\cdot$ & $\cdot \quad \cdot$ & . & 32 & $R_{3,1}$ & $3 S$ & $R_{4,1}$ & $4 S F$ & 123 & & \\
\hline . & . & . & 31 & $R_{3,2}$ & $3-$ & $R_{4,2}$ & $4-$ & 234 & $R_{x, 1}$ & $x S F$ \\
\hline$\cdot$ & $\cdot \quad \cdot$ & $\cdot$ & 34 & $R_{3,3}$ & $3 F S$ & $R_{4,3}$ & $4 F$ & 345 & $R_{x, 2}$ & $x S F$ \\
\hline$B_{x, f_{1}-1}$ & $\rightarrow R_{x, f_{1}-1}$ & $x$ & 24 & & & & & 451 & $R_{x, 3}$ & $x F S$ \\
\hline$B_{x, f_{1}}$ & $\rightarrow R_{x, f_{1}}$ & $x F$ & 14 & & & & & 512 & & \\
\hline & & $S F_{x}$ & & & $S F_{3}$ & & $S F_{4}$ & & & $S F_{x}$ \\
\hline & & & \multirow{2}{*}{\multicolumn{4}{|c|}{$\begin{array}{l}\text { a tight } \operatorname{scccd}(4,2,6) \\
\qquad T_{1}=\{3,4\}\end{array}$}} & \multicolumn{4}{|c|}{$\mathcal{Y}_{3}$} \\
\hline & & & & & & & & \multicolumn{3}{|c|}{$x \in T_{1}=\{1,2,3,4,5\}$} \\
\hline & (a) & & \multicolumn{4}{|c|}{ (b) } & & \multicolumn{3}{|c|}{$(\mathrm{c})$} \\
\hline
\end{tabular}

Fig. 6: $S F$-arrays and examples.

Fig. 6(b) shows a tight $\operatorname{scccd}(4,2,6)$ with elements 3 and $4 \in T_{1}$. The $S F$-array for element $3, S F_{3}$, is shown first; here the $S$ in $R_{3,3}$ appears because element 4 starts in $B_{3,3}$. The array $S F_{4}$ is shown next; here $F \in R_{4,1}$ because 3 finishes in $B_{4,1}$. Fig. 6(c) shows the tight $\operatorname{scccd}(5,3,5), \mathcal{Y}_{3}$; it has $T_{1}=\{1,2,3,4,5\}$ and, for all $x \in T_{1}$, the arrays $S F_{x}$ are identical.

The main idea of this section is to place restrictions on the structure of an $S F$-array of a tight $\operatorname{scccd}(v, k)$ when $v>2 k$. In the following section we 'extend' these $S F$-arrays to tight scccds for $(v, k)=(9,4)$ and $(10,4)$.

So, let us assume that a tight $\operatorname{scccd}(v, k, b), \mathcal{C}$, exists whose set of elements introduced exactly once is $T_{1}$, and let $x \in T_{1}$. Then, using the following Observations (1)-(10), we will establish Criteria (1)-(10) that $S F_{x}$ must satisfy. In the Observations, $R$ denotes an arbitrary row of $S F_{x}$, with corresponding block in $\mathcal{B}_{x}$ denoted by $B_{R}$.

\section{Observations}

(1) Each row $R$ of $S F_{x}$ contains at most one $S$ and at most one $F$. For suppose $R$ contains two or more $S$ s say, then two or more elements are introduced in $B_{R}$, a contradiction. Similarly for the Fs because the reverse of a scced is again a scccd. Clearly the order of the symbols in a row does not matter.

(2) The number of empty rows between any row containing $F$ and the next (different) row containing $S$ as we go down $S F_{x}$ is $\geq b-2 f_{1}+1$. To see this let $y \in T_{1}$ finish in any row of $S F_{x}$ and let $z \in T_{1}$ start in a later one, with $\alpha$ empty rows between them. Now, the pair $\{y, z\}$ is not covered in $\mathcal{B}_{x}$, so the runs containing $y$ and $z$ must meet outside $\mathcal{B}_{x}$. 
That is, $\alpha+f_{\{y\}}+f_{\{z\}} \geq b+1$, so $\alpha \geq b-2 f_{1}+1$.

Furthermore, let $F S(v, k)=b-2 f_{1}+1$. Now $F S(v, k) \geq 0$ with equality if and only if $v=2 k-2$ or $2 k-1$. Now, because we are interested only in $v>2 k$, we may assume that $F S(v, k) \geq 1$, i.e., that there is always at least 1 empty row between a $F$ and the next $S$.

(3) Suppose the three elements $x, y$, and $z \in T_{1}$ (where, without loss of generality, the first is the $x$ of our $S F_{x}$ ) are introduced in three successive blocks in $\mathcal{C}$. See Fig. 7(a) where $y$ starts in $B_{x, 2}$ changing $p$, and $z$ starts in $B_{x, 3}$ changing $q$; see (b) for the $S F$-array so formed. To cover the pairs $\{p, y\}$ and $\{q, z\}$ the elements $p$ and $q$ must occur in the two blocks immediately succeeding $B_{x, f_{1}}$, as shown in (c). Hence, pair $\{p, q\}$ must be in all remaining blocks outside $\mathcal{B}_{x}$, for, if not, it will be covered more than once. So both $p$ and $q \in T_{1}$, which forces $b=2 f_{1}-1$, i.e., $v=2 k-2$ or $2 k-1$. So, with our restriction of $v>2 k$, we may assume that the configurations $\underset{S}{S}$ and $\underset{F}{F}$ do not appear (in $\mathcal{C}$ and so) in $S F_{x}$. (Such a triple $\{x, y, z\}$ is called a persistent triple, it persists through $v-k-1$ blocks, see Phillips and Wallis [1]. Here we have shown that if a tight scccd contains a persistent triple then it must belong to one of the families $\mathfrak{F}$ or $\mathfrak{G}$; see the constructions in $\S 5$ and in the proof of Theorem 6.1; all designs in both these families contain persistent triples.)

\begin{tabular}{|c|c|c|c|c|c|c|}
\hline$B_{x, 1}$ & $x p q$. & $R_{x, 1}$ & $x S$ & $x p q$. & $R_{x, 1}$ & $x S$ \\
\hline$B_{x, 2}$ & $x y q$. & $R_{x, 2}$ & $x S$ & $x y q$. & $R_{x, 2}$ & $x S$ \\
\hline$B_{x, 3}$ & $x y z$. & $R_{x, 3}$ & $x S$ & $x y z$. & . & . \\
\hline . & $\cdots$ & . & . & $\cdots$ & . & . \\
\hline . & $\cdots$ & . & . & $\cdots$ & . & . \\
\hline . & $\cdots$ & . & . & $\cdots$ & $R_{x, f_{1}-1}$ & $x F$ \\
\hline$B_{x, f_{1}}$ & $x y z$. & $R_{x, f_{1}}$ & $x F$ & $x y z$. & $R_{x, f_{1}}$ & $x F$ \\
\hline & $y z \cdot$ & & & $p y z$. & & \\
\hline & $\cdots z \cdot$ & & & $p q z$ & & \\
\hline & $\cdot \cdots$ & & & $\cdots$ & & \\
\hline & $\cdot \cdots$ & & & $\cdots$ & & \\
\hline & (a) & $(\mathrm{b}$ & & $(\mathrm{c})$ & (d) & \\
\hline
\end{tabular}

Fig. 7: Persistent triples and their corresponding forbidden configurations. 
Similarly, the configuration shown in Fig. $7\left(\right.$ d) cannot occur in $S F_{x}$; for, if it did, then the element which starts in $B_{x, 2}$ must finish in the block succeeding $B_{x, f_{1}}$, thus producing the forbidden configuration $\underset{F}{F}$ (in $\mathcal{C}$ ).

(4) The $S$ of $x$ lies in $R_{x, 1}$ and the $F$ in $R_{x, f_{1}}$; and then each of the remaining $t_{1}-1$ elements in $T_{1}$ have either their $S$ or their $F$ present in $S F_{x}$, (but not both, for, if element $y$, say, has both its $S$ and its $F$ present, then its $S$ must appear after its $F$, and so $\{x, y\}$ is covered twice). This gives a total of $t_{1}+1$ symbols.

(5) Consider $R$, an arbitrary row of $S F_{x}$; for each $S$ in or above $R$ there will be an element from $T_{1}$ in $B_{R}$; similarly for each $F$ in or below $R$, except that the $S$ and $F$ of $x$ contribute only one element ( $x$ itself) to $B_{R}$. Now $\mathcal{C}$ has block size $k$, so we must have:

$$
\left\{\begin{array}{c}
\text { the number of } S \mathrm{~s} \\
\text { in or above } R
\end{array}\right\}+\left\{\begin{array}{c}
\text { the number of } F \mathrm{~s} \\
\text { in or below } R
\end{array}\right\}-1 \leq k .
$$

Call the left-hand side of the above equation the weight of $R, w t(R)$; it is the number of elements from $T_{1}$ in $B_{R}$.

(6) Let $R$ be the last row of $S F_{x}$; then $w t(R)$ is the number of $S \mathrm{~s}$ in $S F_{x}$, which is $\leq k$ by Observation (5). Similarly, the number of $F \mathrm{~s}$ in $S F_{x}$ is $\leq k$. Also, using Observation (4), the total number of symbols, $t_{1}+1$, is $\leq 2 k$. Thus, $t_{1} \leq 2 k-1$. There is a tight $\operatorname{scccd}(4,2,6)$ with $\left(t_{1}, t_{2}, t_{3}\right)=(3,0,1)$ for which this inequality is sharp; it is also sharp for any design in the family $\mathfrak{G}$. (Cf. $\S 4$ of $[2]$, where $t_{1} \leq k$.)

(7) Suppose that two adjacent rows in $S F_{x}$ each have weight $k$, then, the corresponding adjacent blocks in $\mathcal{C}$ each contain only elements from $T_{1}$. Let the single-change between these two blocks be caused by $y$ finishing in the first block and $z$ starting in the next. Then, in $S F_{x}$, there are no empty rows between the row containing the $F$ of $y$ and the row containing the $S$ of $z$, a contradiction to Observation (2). Hence, two rows of weight $k$ cannot be adjacent.

(8) The configuration $\underset{S}{S}$ does not occur in $S F_{x}$ so the number of configurations $\underset{S}{S}$ in $S F_{x}$ is $\leq\lfloor k / 2\rfloor$, otherwise the first inequality of Observation (6) is violated. Similarly for the configuration $\underset{F}{F}$.

(9) A persistent pair in $\mathcal{C}$, see [1] and $\S 4$ of [2] , is a pair of elements from $T_{1}$ which start in successive blocks; thus they persist together through $v-k$ blocks. Each persistent pair has a configuration $\underset{S}{S}$ and $\underset{F}{F}$. We claim that our $S F_{x}$ contains exactly one of the configurations $\underset{S}{S}$ or $\underset{F}{F}$ for each persistent pair of $\mathcal{C}$ (except if $\mathcal{C} \in \mathfrak{F}$ or $\mathfrak{G}$ ). 


$$
\begin{gathered}
f_{1}=2\left\{\begin{array}{c}
S \\
S F \\
F
\end{array}\right\} f_{1}=2 \\
f_{1}\left\{\begin{array}{c}
S \\
S \\
\vdots \\
F \\
F
\end{array}\right\} f_{1}
\end{gathered}
$$

$\begin{array}{ll}12 & \\ 32 & 3 S F \\ 31 & 3 F S\end{array}$

$\operatorname{scccd}(3,2,3)$

Fig. 8: Configurations corresponding to a persistent pair.

For any persistent pair $\{y, z\}$ of $\mathcal{C}$ its configurations $\underset{S}{S}$ and $\underset{F}{F}$ can be arranged in one of the two ways shown in Fig. 8(a), where the upper $S$ belongs to $y$. Our claim is clearly true if $x=y$ or $z$, so assume $x \neq y, z$. Now, the $f_{1}$ rows of $S F_{x}$ must include either the $S$ or the $F$ of $y$, and either the $S$ or the $F$ of $z$. That is, they must include either the upper $S$ or the upper $F$ in (a), and either the lower $S$ or the lower $F$, not both in each case. If we choose our $f_{1}$ rows of $S F_{x}$ such that this is true and that neither the whole of the $\underset{S}{S}$ nor the whole of the $\underset{F}{F}$ is included, then, without loss of generality, the first row must be the row containing the lower $F$ and, as it proceeds downwards and cycles around, its last row must be the row containing the upper $S$. Hence, $b=2 f_{1}-1$, i.e., $v=2 k-2$ or $2 k-1$, so $\mathcal{C} \in \mathfrak{F}$ or $\mathfrak{G}$, as in Observation (3). Thus, because $v>2 k$, we may assume that, for any persistent pair of $\mathcal{C}$, our $S F_{x}$ contains either the whole of the pair's $\underset{S}{S}$ or the whole of the pair's $\frac{F}{F}$, but not both.

Now, because each persistent pair in $\mathcal{C}$ contains 2 elements from $T_{1}$, and different persistent pairs contain distinct elements, and $t_{1} \leq 2 k-1$ from Observation (6), we see that the total number of appearances of $\underset{S}{S}$ and $\frac{F}{F}$ in $S F_{x}$ is $\leq\left\lfloor t_{1} / 2\right\rfloor \leq\lfloor(2 k-1) / 2\rfloor=k-1$. (For even $k$ this upper bound is 1 smaller than the upper bound of $2\lfloor k / 2\rfloor$ obtained by adding the upper bounds for the number of appearances of $\underset{S}{S}$ and $\underset{F}{F}$ in Observation (8); for odd $k$ they are the same.)

(10) From Observation (4) $S F_{x}$ contains a total of $t_{1}+1$ symbols, of which at least 1 is $F$. Hence, the number of appearances of $S$ is $\leq t_{1}$. So, via Observation (6), the number of appearances of $S$ is $\leq \min \left\{t_{1}, k\right\}$. Hence, the number of appearances of $F$ is 
$\geq t_{1}+1-\min \left\{t_{1}, k\right\}$; similarly for the number of appearances of $S$. So, finally, we have: $t_{1}+1-\min \left\{t_{1}, k\right\} \leq|S| \leq \min \left\{t_{1}, k\right\}$, and similarly for $|F|$.

So, to summarize our 10 Observations, let $\mathcal{C}$ be an arbitrary tight $\operatorname{scccd}(v, k)$ with $v>2 k$, and let $x \in T_{1}$ and let $R$ be an arbitrary row in $S F_{x}$. Then, corresponding to the 10 Observations above, $S F_{x}$ must satisfy Criteria (1)-(10) below, where $|C|$ denotes the number of appearances of configuration $C$.

\section{Criteria}

(1) $R$ contains at most one $S$ and at most one $F$.

(2) Between any $F$ and the next $S$ there are $\geq F S(v, k)=b-2 f_{1}+1 \geq 1$ empty rows.

(3) The configurations $\underset{S}{S}, \underset{F}{S} \underset{F}{F}$, and the configuration of Fig. $7($ d) do not appear.

(4) $S \in R_{x, 1}, F \in R_{x, f_{1}},|S|+|F|=t_{1}+1$.

(5) $w t(R) \leq k$.

(6) $1 \leq t_{1} \leq 2 k-1$.

(7) Two rows of $w t(k)$ cannot be adjacent.

(8) $|\underset{S}{S}| \leq\lfloor k / 2\rfloor,|\underset{F}{F}| \leq\lfloor k / 2\rfloor$.

(9) $\left|\begin{array}{l}S \\ S\end{array}\right|+\left|\begin{array}{l}F \\ F\end{array}\right| \leq k-1$

(10) $t_{1}+1-\min \left\{t_{1}, k\right\} \leq|S|,|F| \leq \min \left\{t_{1}, k\right\}$.

Finally, some comments relevant to Observation (9).

To see that our claim fails in a design from $\mathfrak{F}$ or $\mathfrak{G}$, consider the tight $\operatorname{scccd}(3,2,3)$ $\mathcal{Y}_{2} \in \mathfrak{G}$ shown in Fig. 8(b). (This corresponds to the second arrangement in (a) where $f_{1}=2$, so $b=3$.) All 3 pairs $\{1,2\},\{1,3\}$, and $\{2,3\}$ are persistent pairs; however $k-1=1$. The array $S F_{3}$ contains the $\underset{S}{S}$ of persistent pair $\{1,3\}$ and the $\underset{F}{F}$ of persistent pair $\{2,3\}$, but neither the $\underset{S}{S}$ nor the $\underset{F}{F}$ of persistent pair $\{1,2\}$. Similarly for all other designs in $\mathfrak{F}$ or $\mathfrak{G}$, where the number of persistent pairs is equal to the number of blocks.

When $v>2 k$, because $S F_{x}$ must contain either the $\underset{S}{S}$ or the $\underset{F}{F}$ of every persistent pair in $\mathcal{C}$, the total number of persistent pairs in $\mathcal{C}$ equals the total number of appearances of $S_{S}$ s and $\underset{F}{F}$ in $S F_{x}$, which is $\leq k-1$ (by Criterion (9)); this upper bound is sharp for the tight $\operatorname{scccd}(9,4,12), \mathcal{U}_{2}$, which contains 3 persistent pairs, see $\S 9$. (Cf. $\S 4$ of $[\underline{2}$ ], where the number of persistent pairs is $\leq k / 2$.) 


\section{Constructions of tight $\operatorname{scccd}(9,4,12) \mathrm{s}$ and $\operatorname{scccd}(10,4,15) \mathrm{s}$ using SF-arrays}

We now illustrate the method of constructing tight designs with $v>2 k$ using $S F$ arrays.

$(\mathbf{v}, \mathbf{k})=(\mathbf{9}, \mathbf{4}) \quad$ First we construct all non-isomorphic tight $\operatorname{scccd}(9,4,12) \mathrm{s}$. To start, we must find all $S F$-arrays for $(v, k)=(9,4)$ that satisfy Criteria (1)-(10) of $\S 8$.

Equation (2) of $\S 7$ gives $A=2, t_{1}+t_{2}=9$, and $t_{1}+2 t_{2}=12$; hence $t_{1}=6$ and $t_{2}=3$. We also have $f_{1}=6$ and $f_{2}=4$. From Criterion $(2), F S(9,4)=1$, i.e., there must be at least 1 empty row between any $F$ and the next $S$ in our $S F$-arrays. Let $T_{1}=\{1,2,3,4,5,6\}$ and $T_{2}=\{7,8,9\}$, and let $x \in T_{1}$.

There are exactly $8 S F$-arrays that satisfy Criteria (1)-(10). Of these, 6 are shown in Fig. 9(a)-(f), and the remaining 2 in (a) and (b) of Fig. 12.

$\begin{array}{lllllll}R_{x, 1} & x S F & x S F & x S F & x S & x S F & x S F \\ R_{x, 2} & x F & x- & x- & x S F & x- & x- \\ R_{x, 3} & x- & x S F & x S & x- & x S F & x S F \\ R_{x, 4} & x S F & x- & x S F & x S F & x- & x F \\ R_{x, 5} & x- & x S & x- & x- & x S F & x- \\ R_{x, 6} & x F S & x F S & x F S & x F S & x F & x F S\end{array}$

(c)

(d)

(e)

Fig. 9: 6 of the $8 S F$-arrays which satisfy Criteria (1)-(10) when $(v, k)=(9,4)$. These 6 form an equivalence class of $S F$-arrays.

In an arbitrary scced $\mathcal{C}$ each of the $t_{1}$ elements of $T_{1}$ has 2 symbols, a $S$ and a $F$; this gives a total of $2 t_{1}$ symbols, of which $t_{1}+1$ appear in $S F_{x}$. We now consider the $t_{1}-1$ 'missing' symbols.

See Fig. 10(a) which contains the $S F_{x}$ of Fig. 9(a). Let us enlarge this $S F_{x}$ from $f_{1}=6$ rows to $b=12$ rows by including the $t_{1}-1=5$ missing symbols and dropping the $x \mathrm{~s}$, see Fig. 10(b). For example, the element which starts in row $R_{x, 4}$ of (a), i.e., in $R_{4}$ of (b), must finish $f_{1}=6$ rows later in $R_{9}$, hence, the $F$ belonging to this element lies in $R_{9}$, as shown. Call this new array with $b$ rows a $S F$-skeleton; note that the $S F$-skeleton (b) is uniquely determined from the $S F_{x}$ in (a).

Now, in Fig. 10(b), let the $S \in R_{1}$ correspond to element 1, the $S \in R_{4}$ correspond to 2, the $S \in R_{6}$ to 3 , the $S \in R_{8}$ to 4 , the $S \in R_{9}$ to 5 , and, finally, the $S \in R_{11}$ to element 6 . 
In the $S F$-skeleton of Fig. 10(b) the $S F$-array $S F_{1}$ (i.e., Fig. 9(a) with $x=1$ ) appears as rows $R_{1}-R_{6}$; also, $S F_{2}$ (Fig. 9 (b) with $x=2$ ) appears as rows $R_{4}-R_{9} ; S F_{3}$ as rows $R_{6}-R_{11} ; S F_{4}$ as rows $R_{8}-R_{1} ; S F_{5}$ as rows $R_{9}-R_{2}$; and, finally, $S F_{6}$ (Fig. $9(\mathrm{f})$ with $x=6$ ) appears as rows $R_{11}-R_{4}$. Thus all 6 of the $S F$-arrays in Fig. 9 occur in the $S F$-skeleton of Fig. 10(b). We say that these $6 S F$-arrays are equivalent $(\sim)$ to one another.

In order to begin extending Fig. 10(b) to a tight scccd(9,4), consider Fig. 10(c), which is a potential tight $\operatorname{scccd}(9,4)$ with all elements from $T_{1}=\{1,2,3,4,5,6\}$ present.

$\begin{array}{lllllllll}R_{x, 1} & x S F & & R_{1} & S F & B_{1} & 1465 & R_{1} & - \\ R_{x, 2} & x F & & R_{2} & F & B_{2} & 1 * 65 & R_{2} & S F \\ R_{x, 3} & x- & \rightarrow & R_{3} & - & B_{3} & 1 * 6 * & R_{3} & - \\ R_{x, 4} & x S F & & R_{4} & S F & B_{4} & 126 * & R_{4} & S F \\ R_{x, 5} & x- & & R_{5} & - & B_{5} & 12 * * & R_{5} & F \\ R_{x, 6} & x F S & & R_{6} & S F & B_{6} & 123 * & R_{6} & - \\ & & R_{7} & - & B_{7} & * 23 * & R_{7} & S F \\ & & R_{8} & S & B_{8} & 423 * & R_{8} & - \\ & & R_{9} & S F & B_{9} & 4235 & R_{9} & S F \\ & & R_{10} & - & B_{10} & 4 * 35 & R_{10} & - \\ & & R_{11} & S F & B_{11} & 4635 & R_{11} & S \\ & & R_{12} & - & B_{12} & 46 * 5 & R_{12} & S F\end{array}$

$S F_{x} \quad S F$-skeleton of $S F_{x}$

(a) (b) reverse of $(b)$

(c) (d)

Fig. 10: An $S F$-array, its $S F$-skeleton, and the corresponding potential tight $\operatorname{scccd}(9,4)$; the reverse of the $S F$-skeleton of $S F_{x}$.

We must now add on the elements in $T_{2}=\{7,8,9\}$; each is introduced twice, and appears in $f_{2}=4$ blocks.

See Fig. 11(a). Without loss of generality start element 7 in $B_{2}$, then $7 \in B_{3}$; for, if not, then both 7 and 5 finish in $B_{2}$, a contradiction. Without loss of generality start 8 in $B_{3}$; this produces (a).

See Fig. 11(b). Now either $7 \in B_{4}$ or $8 \in B_{4}$. If $7 \in B_{4}$ then (because $7 \in T_{2}$ and $\left.f_{2}=4\right) 7$ must start once more in a block containing elements 3 , 4 , and 9 because pairs $\{7,3\},\{7,4\}$, and $\{7,9\}$ will not have been covered, but this is impossible; hence $8 \in B_{4}$. Further, we have (b) by similar reasoning to the above. 
See Fig. $11(\mathrm{c})$. Now if $8 \in B_{7}$, then pair $\{8,9\}$ will be covered twice; so $7 \in B_{7}$ and 7 must occur for 2 successive blocks because $f_{2}=4$ and it has already occurred in 2 blocks. To finish we must have $8 \in B_{10}$ and $9 \in B_{12}$, producing Fig. 11(c).

Let $\mathcal{U}_{1}$ denote this tight $\operatorname{scccd}(9,4,12)$; clearly, by its construction, it is unique up to labelling.

$\begin{array}{lllllllllllllll}B_{1} & 1 & 4 & 6 & 5 & B_{1} & 1 & 4 & 6 & 5 & B_{1} & 1 & 4 & 6 & 5 \\ B_{2} & 1 & 7 & 6 & 5 & B_{2} & 1 & 7 & 6 & 5 & B_{2} & 1 & 7 & 6 & 5 \\ B_{3} & 1 & \mathbf{7} & 6 & \mathbf{8} & B_{3} & 1 & 7 & 6 & 8 & B_{3} & 1 & 7 & 6 & 8 \\ B_{4} & 1 & 2 & 6 & * & B_{4} & 1 & 2 & 6 & \mathbf{8} & B_{4} & 1 & 2 & 6 & 8 \\ B_{5} & 1 & 2 & * & * & B_{5} & 1 & 2 & \mathbf{9} & 8 & B_{5} & 1 & 2 & 9 & 8 \\ B_{6} & 1 & 2 & 3 & B_{6} & 1 & 2 & 3 & \mathbf{9} & B_{6} & 1 & 2 & 3 & 9 \\ B_{7} & * 2 & 3 & B_{7} & * & 2 & 3 & \mathbf{9} & B_{7} & \mathbf{7} & 2 & 3 & 9 \\ B_{8} & 4 & 2 & 3 & B_{8} & 4 & 2 & 3 & * & B_{8} & 4 & 2 & 3 & 7 \\ B_{9} & 4 & 2 & 3 & 5 & B_{9} & 4 & 2 & 3 & 5 & B_{9} & 4 & 2 & 3 & 5 \\ B_{10} & 4 * 3 & 5 & B_{10} & 4 & * & 3 & 5 & B_{10} & 4 & \mathbf{8} & 3 & 5 \\ B_{11} & 4 & 6 & 3 & 5 & B_{11} & 4 & 6 & 3 & 5 & B_{11} & 4 & 6 & 3 & 5 \\ B_{12} & 46 & * & B_{12} & 4 & 6 & * & 5 & B_{12} & 4 & 6 & \mathbf{9} & 5\end{array}$

\section{(a)}

(b)
$\mathcal{U}_{1}, \operatorname{scccd}(9,4,12)$

(c)

Fig. 11: Extending a potential tight $\operatorname{scccd}(9,4)$ to a tight $\operatorname{scccd}(9,4)$.

In general, for a given $(v, k)$ with $v>2 k$, let $\mathcal{S}$ denote the set of $S F$-arrays that satisfy Criteria (1)-(10) and let $S F_{x}$ and $S F_{x}^{\prime}$ be two arbitrary $S F$-arrays in $\mathcal{S}$. We define an equivalence relation $\sim$ on $\mathcal{S}$ as follows:

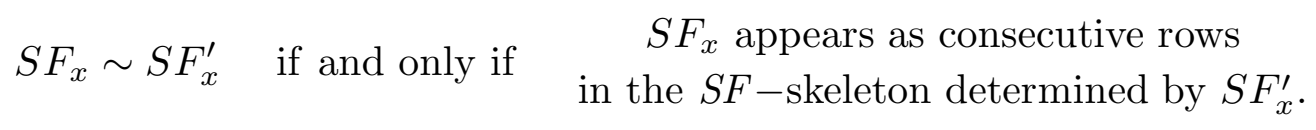

But, because the skeleton determined by an arbitrary $S F$-array in $\mathcal{S}$ is unique, we can redefine $\sim$ as:

$$
\begin{array}{r}
\text { the } S F \text {-skeleton determined by } S F_{x} \sim S F_{x}^{\prime} \quad \text { if and only if } \quad \text { is a cyclic shift of the } S F \text {-skeleton determined by } S F_{x}^{\prime} .
\end{array}
$$

It is straightforward to prove that $\sim$ is an equivalence relation.

Each equivalence class of $S F$-arrays gives rise to one $S F$-skeleton; we say that this $S F$ skeleton represents the class. Instead of attempting to extend all $S F$-arrays (or, rather, 
their $S F$-skeletons) from a particular equivalence class to tight designs, we need only attempt to extend the $S F$-skeleton that represents this class. (Also, if two $S F$-skeletons represent different classes, and both can be extended to tight designs, then these designs are non-isomorphic.) However, a single $S F$-skeleton can be extended to non-isomorphic designs, see Fig. 14.

The remaining $2 S F$-arrays for $(v, k)=(9,4)$ are shown in (a) and (b) of Fig. 12; they form another equivalence class. The $S F$-skeleton of (b) is (c) which extends uniquely (up to labelling) to the tight $\operatorname{scccd}(9,4,12)$ shown in (d); call it $\mathcal{U}_{2}$. The $S F$-arrays (a) and (b) each occur 3 times each amongst the $t_{1}=6 S F$-arrays of $\mathcal{U}_{2}$. This tight design contains $k-1=3$ persistent pairs, which, by the comments at the end of $\S 8$, is the maximum number allowed in a tight $\operatorname{scccd}(v, k)$ with $v>2 k$.

\begin{tabular}{|c|c|c|c|c|c|c|c|}
\hline$R_{x, 1}$ & $x S F$ & $x S$ & & $R_{1}$ & $S$ & $B_{1}$ & 1956 \\
\hline$R_{x, 2}$ & $x F$ & $x S F$ & & $R_{2}$ & $S F$ & $B_{2}$ & 1256 \\
\hline$R_{x, 3}$ & $x-$ & $x F$ & $\rightarrow$ & $R_{3}$ & $F$ & $B_{3}$ & 1276 \\
\hline$R_{x, 4}$ & $x S$ & $x-$ & & $R_{4}$ & - & $B_{4}$ & 1278 \\
\hline$R_{x, 5}$ & $x S F$ & $x S$ & & $R_{5}$ & $S$ & $B_{5}$ & 1238 \\
\hline \multirow[t]{9}{*}{$R_{x, 6}$} & $x F$ & $x F S$ & & $R_{6}$ & $S F$ & $B_{6}$ & 1234 \\
\hline & & & & $R_{7}$ & $F$ & $B_{7}$ & 9234 \\
\hline & & & & $R_{8}$ & - & $B_{8}$ & 9734 \\
\hline & & & & $R_{9}$ & $S$ & $B_{9}$ & 5734 \\
\hline & & & & $R_{10}$ & $S F$ & $B_{10}$ & 5634 \\
\hline & & & & $R_{11}$ & $F$ & $B_{11}$ & 5684 \\
\hline & & & & $R_{12}$ & - & $B_{12}$ & 5689 \\
\hline & & & & eleto & of $(b)$ & $\mathcal{U}_{2}, \operatorname{scc}$ & $\operatorname{cd}(9,4,12)$ \\
\hline & (a) & (b) & & & & & $(\mathrm{d})$ \\
\hline
\end{tabular}

Fig. 12: The remaining $2 S F$-arrays for $(v, k)=(9,4)$, which form another equivalence class; the $S F$-skeleton which represents this class and the corresponding tight $\operatorname{scccd}(9,4)$.

Now, because the $S F$-skeletons from which $\mathcal{U}_{1}$ and $\mathcal{U}_{2}$ were formed represent different equivalence classes, we have $\mathcal{U}_{1} \nsucceq \mathcal{U}_{2}$. This gives us:

Theorem 9.1. There are 2 non-isomorphic tight $\operatorname{scccd}(9,4,12)$ s, namely $\mathcal{U}_{1}$ and $\mathcal{U}_{2}$ shown above. 
As mentioned at the end of $\S 4$, the reverse of $\operatorname{acccd}(v, k, b), \mathcal{B}$, is another $\operatorname{scccd}(v, k, b)$, $\operatorname{rev}(\mathcal{B})$; and, if a scccd $\mathcal{C}$ is tight then $\operatorname{rev}(\mathcal{C})$ is also tight. Hence, from Theorem 9.1, $\operatorname{rev}\left(\mathcal{U}_{1}\right) \cong \mathcal{U}_{1}$ or $\mathcal{U}_{2}$.

For an arbitrary scccd $\mathcal{C}$, to obtain the $S F$-skeleton of $\operatorname{rev}(\mathcal{C})$ from the $S F$-skeleton of $\mathcal{C}$ we reverse the order of its rows and switch $S \leftrightarrow F$.

The $S F$-skeleton of $\mathcal{U}_{1}$ is shown in Fig. 10(b) and the $S F$-skeleton of $\operatorname{rev}\left(\mathcal{U}_{1}\right)$ in Fig. 10(d); it is a cyclic shift of the $S F$-skeleton of $\mathcal{U}_{1}$. Hence, because the extension of the $S F$-skeleton of $\mathcal{U}_{1}$ to a tight design is unique up to labelling, we have $\operatorname{rev}\left(\mathcal{U}_{1}\right) \cong \mathcal{U}_{1}$. Thus $\mathcal{U}_{1}$ and $\mathcal{U}_{2}$ are self-reverse.

$(\mathbf{v}, \mathbf{k})=(\mathbf{1 0}, \mathbf{4}) \quad$ We now construct all non-isomorphic tight $\operatorname{scccd}(10,4,15) \mathrm{s}$.

Equation (2) of $\S 7$ yields the three solutions: $\left(t_{1}, t_{2}, t_{3}\right)=(7,1,2),(6,3,1)$, and $(5,5,0)$. Here $f_{3}=3$, so Lemma 7.1(iii) with $j=3$ disposes of the first solution. For the remaining two let $\mathcal{V}$ be a tight $\operatorname{scccd}(10,4,15)$.

(i) $\left(t_{1}, t_{2}, t_{3}\right)=(6,3,1)$. We could use $S F$-arrays here but, for variety, we prefer the following approach which is justified by the result: there are 2 non-isomorphic tight $\operatorname{scccd}(10,4,15)$ s, both of which can be constructed by 'expanding' $\mathcal{U}_{2}$ of Fig. $12(\mathrm{~d})$.

First some definitions, see $\S 7$ of [2] . For any $\operatorname{scccd}(v, k, b), \mathcal{B}=\left\{B_{1}, \ldots, B_{b}\right\}$, and for any $i=1, \ldots, b-1$ let $U_{i}$ be the subset of $k-1$ elements which survives from $B_{i}$ to $B_{i+1}$; we call $U_{i}$ the unchanged subset at location $i$. Also, let $U_{b}$ be the subset of $k-1$ elements which survives from $B_{b}$ to $B_{1}$, the unchanged subset at location $b$.

As before, let $T_{1}=\{1,2,3,4,5,6\}$ and $T_{2}=\{7,8,9\}$, and so $T_{3}=\{10\}$. Now, because $f_{3}=3$, each time element 10 is introduced into a block it is immediately changed. So the arrangement of blocks shown below must occur 3 times, at the pairs of consecutive locations: $\ell_{1}, \ell_{1}+1$, and $\ell_{2}, \ell_{2}+1$, and $\ell_{3}, \ell_{3}+1$. We have shown the arrangement at the pair of locations $\ell, \ell+1$ for any $\ell \in\left\{\ell_{1}, \ell_{2}, \ell_{3}\right\}$.

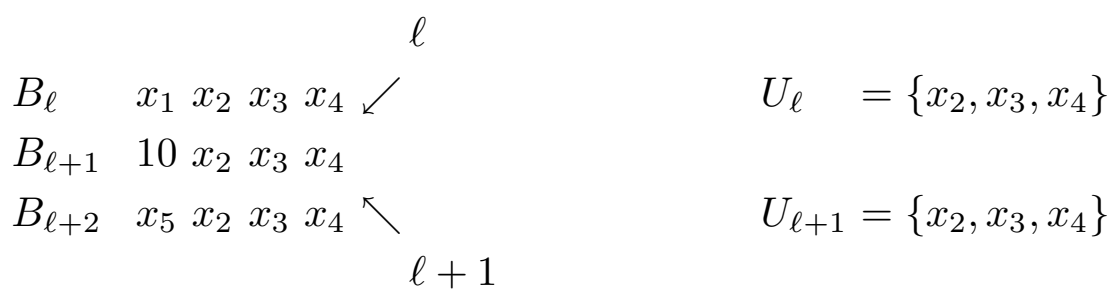

Here $U_{\ell}=U_{\ell+1}$, i.e., the two unchanged subsets for this arrangement of blocks are equal. Hence, each of the 3 pairs of locations produces an unchanged subset which survives through the pair of locations; and, because $\mathcal{V}$ is tight, these 3 unchanged subsets partition the set $\{1,2, \ldots, 9\}$. 
Now if we remove the 3 blocks $B_{\ell_{1}+1}, B_{\ell_{2}+1}$, and $B_{\ell_{3}+1}$ that contain element 10 from $\mathcal{V}$ we obtain a tight $\operatorname{scccd}(9,4,12)$ with 3 unchanged subsets which partition $\{1,2, \ldots, 9\}$, i.e., with an expansion set of locations, see $\S 7$ of [2]. Of $\mathcal{U}_{1}$ and $\mathcal{U}_{2}$, only $\mathcal{U}_{2}$ has an expansion set of locations, in fact it has two:

$$
\begin{array}{ll}
\{1,2,7\} \text { at location } 3, & \{1,2,8\} \text { at location } 4, \\
\{9,3,4\} \text { at location } 7, & \text { and } \quad\{7,3,4\} \text { at location } 8, \\
\{5,6,8\} \text { at location } 11, & \{5,6,9\} \text { at location } 12 .
\end{array}
$$

Expanding $\mathcal{U}_{2}$ at the first expansion set above with element 10 gives us the tight

\begin{tabular}{|c|c|c|c|c|c|c|c|}
\hline$B_{1}$ & 1956 & $S$ & $S_{1} F_{2}$ & $B_{1}$ & $\begin{array}{llll}1 & 9 & 5 & 6\end{array}$ & $S$ & $S_{1} F_{2}$ \\
\hline$B_{2}$ & 1256 & $S F$ & $S_{1} F_{1}$ & $B_{2}$ & 1256 & $S F$ & $S_{1} F_{1}$ \\
\hline$B_{3}$ & 1276 & $F$ & $S_{2} F_{1}$ & $B_{3}$ & $\begin{array}{llll}1 & 2 & 7 & 6\end{array}$ & $F$ & $S_{2} F_{1}$ \\
\hline$B_{4}$ & 12710 & - & $S_{3} F_{3}$ & $B_{4}$ & $\begin{array}{llll}1 & 2 & 7 & 8\end{array}$ & - & $S_{2} F_{2}$ \\
\hline$B_{5}$ & 1278 & - & $S_{2} F_{2}$ & $B_{5}$ & 12108 & - & $S_{3} F_{3}$ \\
\hline$B_{6}$ & 1238 & $S$ & $S_{1} F_{2}$ & $B_{6}$ & 1238 & $S$ & $S_{1} F_{2}$ \\
\hline$B_{7}$ & 1234 & $S F$ & $S_{1} F_{1}$ & $B_{7}$ & $\begin{array}{llll}1 & 2 & 3 & 4\end{array}$ & $S F$ & $S_{1} F_{1}$ \\
\hline$B_{8}$ & $\begin{array}{llll}9 & 2 & 3 & 4\end{array}$ & $F$ & $S_{2} F_{1}$ & $B_{8}$ & $\begin{array}{llll}9 & 2 & 3 & 4\end{array}$ & $F$ & $S_{2} F_{1}$ \\
\hline$B_{9}$ & 91034 & - & $S_{3} F_{3}$ & $B_{9}$ & $\begin{array}{llll}9 & 7 & 3 & 4\end{array}$ & - & $S_{2} F_{2}$ \\
\hline$B_{10}$ & $\begin{array}{llll}9 & 7 & 3 & 4\end{array}$ & - & $S_{2} F_{2}$ & $B_{10}$ & 10734 & - & $S_{3} F_{3}$ \\
\hline$B_{11}$ & $\begin{array}{llll}5 & 7 & 3 & 4\end{array}$ & $S$ & $S_{1} F_{2}$ & $B_{11}$ & $\begin{array}{llll}5 & 73 & 4\end{array}$ & $S$ & $S_{1} F_{2}$ \\
\hline$B_{12}$ & $\begin{array}{llll}5 & 6 & 3 & 4\end{array}$ & $S F$ & $S_{1} F_{1}$ & $B_{12}$ & $\begin{array}{llll}5 & 6 & 3 & 4\end{array}$ & $S F$ & $S_{1} F_{1}$ \\
\hline$B_{13}$ & $\begin{array}{llll}5 & 6 & 8 & 4\end{array}$ & $F$ & $S_{2} F_{1}$ & $B_{13}$ & $\begin{array}{llll}5 & 6 & 8 & 4\end{array}$ & $F$ & $S_{2} F_{1}$ \\
\hline$B_{14}$ & $\begin{array}{lll}5 & 6 & 810\end{array}$ & - & $S_{3} F_{3}$ & $B_{14}$ & $\begin{array}{llll}5 & 6 & 8 & 9\end{array}$ & - & $S_{2} F_{2}$ \\
\hline$B_{15}$ & $\begin{array}{llll}5 & 6 & 8 & 9\end{array}$ & - & $S_{2} F_{2}$ & $B_{15}$ & 56109 & - & $S_{3} F_{3}$ \\
\hline
\end{tabular}
$\operatorname{scccd}(10,4,15), \mathcal{V}_{1}$ shown in Fig. 13(a); and, $\mathcal{V}_{2}$ in (b) comes from using the second expansion set.

(a)

(b)

Fig. 13: The 2 non-isomorphic tight $\operatorname{scccd}(10,4) \mathrm{s}$ with $\left(t_{1}, t_{2}, t_{3}\right)=(6,3,1)$, their $S F$ skeletons and complete $S F$-skeletons. Both of these designs come from expanding $\mathcal{U}_{2}$ of Fig. 12(d).

Now we show that $\mathcal{V}_{1}$ and $\mathcal{V}_{2}$ are non-isomorphic even though their $S F$-skeletons are cyclic shifts of each other. 
See Fig. 13. Consider the complete $S F$-skeleton shown to the right of the $S F$-skeletons. In any block of a scccd one element starts and one finishes. Suppose that an element from $T_{j}$ starts and that one from $T_{j^{\prime}}$ finishes, then the corresponding row of the complete $S F$-skeleton is $S_{j} F_{j^{\prime}}$. Thus, the complete $S F$-skeleton includes start-finish information about all elements in $[v]$, not just those in $T_{1}$. Clearly, if two designs are isomorphic, then their complete $S F$-skeletons must be cyclic shifts of one another; this is not so for $\mathcal{V}_{1}$ and $\mathcal{V}_{2}$, hence $\mathcal{V}_{1} \neq \mathcal{V}_{2}$. So there are exactly 2 non-isomorphic tight $\operatorname{scccd}(10,4,15) \mathrm{s}$ with $\left(t_{1}, t_{2}, t_{3}\right)=(6,3,1)$, namely $\mathcal{V}_{1}$ and $\mathcal{V}_{2}$.

The reverse of a tight $\operatorname{scccd}(v, k), \mathcal{C}$, is another tight $\operatorname{scccd}(v, k), \operatorname{rev}(\mathcal{C})$. Moreover, for $j=1, \ldots, A$, we have equality amongst the sets $T_{j}$ for $\mathcal{C}$ and $\operatorname{rev}(\mathcal{C})$, and so equality amongst the numbers $t_{j}$ for $\mathcal{C}$ and $\operatorname{rev}(\mathcal{C})$.

So $\operatorname{rev}\left(\mathcal{V}_{1}\right)$ also has $\left(t_{1}, t_{2}, t_{3}\right)=(6,3,1)$, and thus $\operatorname{rev}\left(\mathcal{V}_{1}\right) \cong \mathcal{V}_{1}$ or $\mathcal{V}_{2}$. Now if $\operatorname{rev}\left(\mathcal{V}_{1}\right) \cong \mathcal{V}_{1}$, then the complete $S F$-skeleton of $\operatorname{rev}\left(\mathcal{V}_{1}\right)$ must be a cyclic shift of the complete $S F$-skeleton of $\mathcal{V}_{1}$, but this is not the case. Hence, $\operatorname{rev}\left(\mathcal{V}_{1}\right) \cong \mathcal{V}_{2}$. So $\mathcal{V}_{1}$ is not isomorphic to its reverse, similarly for $\mathcal{V}_{2}$.

For two scccds, $\mathcal{B}$ and $\mathcal{B}^{\prime}$, we write $\mathcal{B}$ r $\mathcal{B}^{\prime}$ if $\mathcal{B} ¥ \mathcal{B}^{\prime}$, but $\operatorname{rev}(\mathcal{B}) \cong \mathcal{B}^{\prime}$ (or, equivalently, $\left.\operatorname{rev}\left(\mathcal{B}^{\prime}\right) \cong \mathcal{B}\right)$. Thus $\mathcal{V}_{1} \mathrm{r} \mathcal{V}_{2}$

(ii) $\left(t_{1}, t_{2}, t_{3}\right)=(5,5,0)$. There are $32 S F$-arrays that satisfy Criteria (1)-(10), and 8 equivalence classes of $S F$-arrays, 6 of size 5 and 2 of size 1 .

The $S F$-skeletons of the 8 classes are shown in Fig. 14; underneath each is the number of its extensions to non-isomorphic designs, and the names of the designs.

So there are 10 non-isomorphic tight $\operatorname{scccd}(10,4) \mathrm{s}$ with $\left(t_{1}, t_{2}, t_{3}\right)=(5,5,0)$, namely $\mathcal{V}_{m}$ for $3 \leq m \leq 12$. We also have: $\mathcal{V}_{3} \mathrm{r} \mathcal{V}_{5}, \mathcal{V}_{4} \mathrm{r} \mathcal{V}_{6}, \mathcal{V}_{7} \mathrm{r} \mathcal{V}_{8}, \mathcal{V}_{9} \mathrm{r} \mathcal{V}_{10}$, and $\mathcal{V}_{11} \mathrm{r} \mathcal{V}_{12}$

The 2 designs from the previous case give us:

Theorem 9.2. There are 12 non-isomorphic tight $\operatorname{scccd}(10,4) \mathrm{s}$, namely $\mathcal{V}_{m}$ for $m=$ $1, \ldots, 12$. 


$\begin{array}{clllllll}S F & - & S F & - & S F & F & S F & S F \\ F & - & F & F & F & - & F & - \\ - & S F & - & - & - & - & - & - \\ F & - & - & - & - & - & - & S F \\ - & F & S F & S F & S & S F & - & - \\ - & F & - & F & F & F & S & - \\ S F & - & F & - & F & - & S F & S F \\ - & - & - & - & - & - & - & - \\ - & S F & - & S & - & S & - & - \\ S & - & S & - & S & S & S & S F \\ S & - & S F & S F & S F & F & S & - \\ - & S & - & - & - & - & F & - \\ S F & - & - & - & - & - & F & S F \\ - & S & S & S & - & S & - & - \\ - & S F & - & S F & S & S F & - & - \\ & & & & & & & \\ 2 & 2 & 1 & 1 & 1 & 1 & 0 & 2 \\ \mathcal{V}_{3}, \mathcal{V}_{4} & \mathcal{V}_{5}, \mathcal{V}_{6} & \mathcal{V}_{7} & \mathcal{V}_{8} & \mathcal{V}_{9} & \mathcal{V}_{10} & & \mathcal{V}_{11}, \mathcal{V}_{12}\end{array}$

Fig. 14: The $8 S F$-skeletons which represent the 8 equivalence classes of $S F$-arrays for $(v, k)=(10,4)$ and $\left(t_{1}, t_{2}, t_{3}\right)=(5,5,0)$. Underneath each $S F$-skeleton is the number of its extensions to non-isomorphic designs, and the names of the designs.

\section{Non-existence of some tight designs}

In this section we consider three parameter sets for $(v, k)$ :

(i) $\{(3 k-3, k): k$ even and $\geq 2\}$;

(ii) $\{(3 k-2, k): k$ even and $\geq 2\}$;

(iii) $\left\{\left((i+1)^{2} / 4,\left(i^{2}+7\right) / 8\right): i\right.$ odd and $\left.\geq 3\right\}$.

Every $(v, k)$ in (i), (ii), and (iii) above satisfies the division requirement $2(k-1) \mid v(v-1)$ for a tight design to exist; however, for (i) and (ii), tight designs only exist when $k=2$ or 4 , and, for (iii), only when $i=3$ or 5 .

Using the notation of [ $[\underline{]}$, we denote by $S C D(v, k, b)$ a single-change (non-circular) covering design on $[v]$ with $b$ blocks of size $k$. We let $f(v, k)$ be the smallest $b$ for which there exists a $S C D(v, k, b)$. The function $f(v, k)$ is studied in [ [5], []] , and []] .

Now a $\operatorname{scccd}(v, k, b)$ is also a $S C D(v, k, b)$. In particular, a minimal $\operatorname{scccd}\left(v, k, b_{*}(v, k)\right)$ is a $S C D\left(v, k, b_{*}(v, k)\right)$, so we have $f(v, k) \leq b_{*}(v, k)$. (There are many $(v, k)$ s for which 
equality holds.)

In $\S \S 5$ and 6 we considered $\mathfrak{F}$ and $\mathfrak{G}$, two families of tight $\operatorname{scccd}(v, k) \mathrm{s}$ for $v=2 k-2$ and $2 k-1$ respectively. In the following two theorems we consider $v=3 k-3$ (case (i) above) and $3 k-2$ ((ii) above) respectively.

Theorem 10.1. A tight $\operatorname{scccd}(3 k-3, k,(9 k-12) / 2)$ exists only when $k=2$ or 4 .

Proof. Here $k$ is even. Consider the pair $(3 k-3, k)$ for $k \geq 6$, from Theorem 3.3 of [ $\underline{5}]$ we have $f(3 k-3, k)=5 k-8$. If a tight $\operatorname{scccd}(3 k-3, k,(9 k-12) / 2)$ exists then we must have $5 k-8 \leq(9 k-12) / 2$, a contradiction. Thus a tight $\operatorname{scccd}(3 k-3, k)$ does not exist for $k \geq 6$.

For $k=2$ we have a tight $\operatorname{scccd}(3,2,3), \mathcal{Y}_{2}$, and for $k=4$ a tight $\operatorname{scccd}(9,4,12)$, e.g., $\mathcal{U}_{1}$.

So we have infinitely many pairs $(v, k)=(3 k-3, k)$ where $k$ is even and $\geq 6$, for which $2(k-1) \mid v(v-1)$ but a tight $\operatorname{scccd}(v, k)$ does not exist, e.g., a tight $\operatorname{scccd}(15,6,21)$ does not exist.

Similarly for $v=3 k-2$ :

Theorem 10.2. A tight $\operatorname{scccd}(3 k-2, k,(9 k-6) / 2)$ exists only when $k=2$ or 4 .

When $k=2$ we have a tight $\operatorname{scccd}(4,2,6)$ and $k=4$ a tight $\operatorname{scccd}(10,4,15)$, e.g., $\mathcal{V}_{1}$. Theorems 10.1 and 10.2 can also be proved using $S F$-arrays.

The final result in this section, Theorem 10.4, will, for variety and interest, be proved using the following lemma, although it can also be proved in a similar manner to the above.

Lemma 10.3. Let $\mathcal{C}$ be a tight $\operatorname{scccd}(v, k)$ with $v>2 k, x \in T_{1}, t_{1}=\left|T_{1}\right|$, and $f_{1}=$ $v-k+1$. Then

(i) the total number of symbols in any $r$ successive rows of $S F_{x}$ is $\leq r+1$;

(ii) $t_{1} \leq f_{1}$.

Proof.

(i) A straightforward proof by induction on $r$.

(ii) For any $x \in T_{1}$, the $S F$-array $S F_{x}$ has $f_{1}$ rows, hence $\leq f_{1}+1$ symbols. But, by Criterion (4), it has exactly $t_{1}+1$ symbols. Hence $t_{1} \leq f_{1}$.

The inequality $t_{1} \leq f_{1}$ is sharp for both of the tight $\operatorname{scccd}(9,4,12) \operatorname{s} \mathcal{U}_{1}$ and $\mathcal{U}_{2}$ of $\S 9$.

Let $\mathcal{C}$ be a tight $\operatorname{scccd}(v, k)$. From Observation (2) we have $2 f_{1} \leq b+1$, with equality if and only if $\mathcal{C} \in \mathfrak{F}$ or $\mathfrak{G}$. So, for tight designs other than those in $\mathfrak{F}$ or $\mathfrak{G}$, we have $2 f_{1} \leq b$. 
We now classify tight designs with $2 f_{1}=b$, so $v \geq 2 k$.

Theorem 10.4. A tight $\operatorname{scccd}(v, k, b)$ with $2 f_{1}=b$ is a tight $\operatorname{scccd}(4,2,6)$ or a tight $\operatorname{scccd}(9,4,12)$.

Proof. $\quad$ Let $\mathcal{D}$ be a tight $\operatorname{scccd}(v, k)$ with $2 f_{1}=b$ and $v \geq 2 k$.

We have $2(v-k+1)=\frac{v(v-1)}{2(k-1)}$, i.e., $v=(4 k-3+\sqrt{(8 k-7)}) / 2$. So let $k=\left(i^{2}+7\right) / 8$ where $i$ is odd and $\geq 3$. Hence, $(v, k)=\left((i+1)^{2} / 4,\left(i^{2}+7\right) / 8\right)$ ((iii) above), and $f_{1}=$ $\left(i^{2}+4 i+3\right) / 8$.

Equation $(2)$ from $\S 7$ then gives $f_{3}=(v-1)-3(k-2)=\left(21+4 i-i^{2}\right) / 8$. So, for $i \geq 7$, we have $f_{3} \leq 0<3$. Thus, from Lemma 7.1(ii) with $j=3$, we have $t_{\ell}=0$ for $\ell \geq 3$, i.e., every element in $\mathcal{D}$ is introduced once or twice. So, equation (2) yields:

$$
t_{1}+t_{2}=v=\frac{(i+1)^{2}}{4} ; \quad t_{1}+2 t_{2}=b=\frac{\left(i^{2}+4 i+3\right)}{4} .
$$

This gives $t_{1}=\left(i^{2}-1\right) / 4$. Now, for $i \geq 7$ we have $v>2 k$, so, via Lemma 10.3(ii), we must have $t_{1} \leq f_{1}$; but this is false when $i \geq 7$. Thus, a tight $\operatorname{scccd}\left((i+1)^{2} / 4,\left(i^{2}+7\right) / 8\right)$ does not exist for $i \geq 7$.

For $i=3$ a tight $\operatorname{scccd}(4,2,6)$ exists and for $i=5$ a tight $\operatorname{scccd}(9,4,12)$ exists.

\section{Perfect designs; column-regular designs; element-regular designs}

Again, let $\mathcal{B}=\left\{B_{1}, \ldots, B_{b}\right\}$ be an arbitrary $\operatorname{scccd}(v, k, b)$, and, for each $i=1, \ldots, b$, let $\operatorname{sf}\left(\mathcal{B}_{i}\right)$ be the standardized form of its $i$-th cyclic shift $\mathcal{B}_{i}$, see $\S 4$.

Now consider $\operatorname{sf}\left(\mathcal{B}_{i}\right)$ for any fixed $i=1, \ldots, b$; its first block is $(1,2, \ldots, k)$. For $r=1, \ldots, k$, let its $r$-th column be the column beginning with $r$, and let $\eta_{i, r}$ be the number of elements introduced into this column. Now let $A_{i}$ be the ordered $k$-tuple $\left[\eta_{i, 1}, \ldots, \eta_{i, k}\right]$; call this the column-array of $\operatorname{sf}\left(\mathcal{B}_{i}\right)$.

Consider again $\mathcal{E}=\left\{B_{1}, \ldots, B_{8}\right\}$, the $\operatorname{scccd}(6,3,8)$ from Fig. 3(a) and its standardized form $\operatorname{sf}\left(\mathcal{E}_{1}\right)=\left\{L_{1}, \ldots, L_{8}\right\}$ from Fig. 3(b) shown again in Fig. 15(a); we have $A_{1}=$ $[3,2,3]$. Now consider Fig. 15(b), which shows $\operatorname{sf}\left(\mathcal{E}_{5}\right)$, the standardized form of $\mathcal{E}_{5}=$ $\left\{B_{5}, B_{6}, B_{7}, B_{8}, B_{1}, B_{2}, B_{3}, B_{4}\right\}$, this design has $A_{5}=[1,4,3]$. So, for a fixed $\mathcal{B}$, we may have different $A_{i}$ for different $i$. 
$L_{1} 123$

123

734

43

$L_{2} \quad 124$

124

735

53

$L_{3} \quad 154$

154

835

52

$L_{4} 164$

152

635

51

$L_{5} \quad 364$

652

625

123

41

$L_{6} \quad 365$

653

628

124

45

$L_{7} \quad 265$

643

128

154

42

$L_{8} \quad 263$

613

127

354

32

$\operatorname{sf}(\mathcal{E})$

$\operatorname{sf}\left(\mathcal{E}_{5}\right)$

$\operatorname{scccd}(8,3,14)$

$A_{1}=[3,2,3]$

(a)

$A_{2}=[1,4,3]$

(b)

(c)

(d)

(e)

(c) the representative standardized form of a perfect $\operatorname{scccd}(8,3,14)$ : each $A_{i}$ is a permutation of $A_{1}=[4,5,5]$,

(d) $\operatorname{rsf}\left(\mathcal{Y}_{3}\right)$ : each $A_{i}$ is a permutation of $A_{1}=[2,1,2]$, not perfect, element-regular with $\mu=1$,

(e) $\operatorname{scccd}(5,2,10)$ : column-regular with $\eta=5$, perfect, element-regular with $\mu=2$.

Fig. 15: Standardized designs and their column-arrays, and other properties.

A standardized $\operatorname{scccd}(v, k, b) \mathcal{B}=\left\{B_{1}, \ldots, B_{b}\right\}$ is perfect if each of the unchanged elements between $B_{b}$ and $B_{1}$ is in the same column in $B_{b}$ as in $B_{1}$. So, the two ends of a perfect standardized scccd can be 'joined-up' to give a circular version of requirement $(4)$ in the definition of standardization $(\S 4)$. For any $\mathcal{B}$, all of its $b$ standardized forms are perfect or none are. Hence, a standardized $\mathcal{B}$ is perfect if and only if $\operatorname{rsf}(\mathcal{B})$ is perfect. The standardized form of $\mathcal{Y}_{3}$ (from Fig. 1(b)) is shown in Fig. 15(d); this is also $\operatorname{rsf}\left(\mathcal{Y}_{3}\right)$, it is not perfect. See Fig. 15(c) for the representative standardized form of a perfect $\operatorname{scccd}(8,3,14)$. 
An interesting property of perfect standardized scccds is:

\section{Theorem 11.1.}

Let $A_{1}, \ldots, A_{b}$ be the column-arrays of a perfect standardized $\operatorname{scccd}(v, k, b)$. Then each $A_{i}$ is a permutation of $A_{1}$, for $i=1, \ldots, b$.

Proof. For any fixed $r=1, \ldots, k$, consider the $r$-th column in a perfect standardized $\operatorname{scccd}(v, k, b) \mathcal{B}=\operatorname{sf}\left(\mathcal{B}_{1}\right)=\left\{B_{1}, \ldots, B_{b}\right\}$. The elements in this column in $B_{b}$ and $B_{1}$ are either the same, or different if the single-change between $B_{b}$ and $B_{1}$ occurs in this column. In either case, we may write the elements of this column in a circle. Then $\eta_{1, r}$, the number of introductions in this column, is counted starting at $B_{1}$; this number is fixed no matter where on the circle we start. Now let $\operatorname{sf}\left(\mathcal{B}_{2}\right)$ be formed from $\mathcal{B}_{2}=\left\{B_{2}, B_{3}, \ldots, B_{b}, B_{1}\right\}$ by a permutation of $[v]$ and a permutation $\phi$ of $[k]$, i.e., of the columns. Then $\eta_{2, r}$, the number of introductions in column $r$ of $\operatorname{sf}\left(\mathcal{B}_{2}\right)$, is equal to the number of introductions in column $\phi^{-1}(r)$ of $\operatorname{sf}\left(\mathcal{B}_{1}\right)$ when starting counting at $B_{2}$, which is the same as starting at $B_{1}$; this number is $\eta_{1, \phi^{-1}(r)}$. Thus $\eta_{2, r}=\eta_{1, \phi^{-1}(r)}$ for $r=1, \ldots, k$. That is, $A_{2}$ is a permutation of $A_{1}$, and so on for $A_{i}, i=3, \ldots, b$.

For example, in the perfect standardized $\operatorname{scccd}(8,3,14)$ in Fig. 15(c), each $A_{i}$ is a permutation of $A_{1}=[4,5,5]$. The $\operatorname{scccd}(5,3,5)$ shown in Fig. $15(\mathrm{~d})$ is $\operatorname{rsf}\left(\mathcal{Y}_{3}\right)$. Each column-array of this design is a permutation of $A_{1}=[2,1,2]$, even though it is not perfect, so the converse of Theorem 11.1 is not true.

Consider $\operatorname{sf}\left(\mathcal{B}_{i}\right)$ for a fixed $i$, if the number of introductions into each column is the same, then we say that $\operatorname{sf}\left(\mathcal{B}_{i}\right)$ is column-regular, see $\S 4$ of [2] . So $\eta_{i, r}=\eta=b / k$ for each $r=1, \ldots, k$, and $A_{i}=[\eta, \ldots, \eta]$. Also, $\mathcal{B}$ itself is column-regular if $\operatorname{sf}\left(\mathcal{B}_{i}\right)$ is column-regular for each $i=1, \ldots, b$. So a $\operatorname{sccd}(v, k, b)$ is column-regular if each of its $b$ standardized forms is itself column-regular.

Although we have defined a column-array only for a standardized scced we can also define it for a column-strict scced. So, the column-array of a column-strict $\operatorname{scccd}(v, k, b)$, $\mathcal{B}$, is the ordered $k$-tuple $\left[\eta_{1}, \ldots, \eta_{k}\right]$ where $\eta_{r}$ is the number of elements introduced into the $r$-th column of $\mathcal{B}$, for each $r=1, \ldots, k$.

The definitions of 'perfect' and 'column-regular' can also be carried over to columnstrict scccds; and a $\operatorname{scccd}(v, k, b), \mathcal{B}=\left\{B_{1}, \ldots, B_{b}\right\}$, is column-regular if the column-strict representation of each $\mathcal{B}_{i}$ is itself column-regular.

We can now prove:

Theorem 11.2. A standardized column-regular $\operatorname{scccd}(v, k, b)$ is perfect.

Proof. Let $\mathcal{B}=\operatorname{sf}\left(\mathcal{B}_{1}\right)=\left\{B_{1}, \ldots, B_{b}\right\}$ be a standardized column-regular $\operatorname{scccd}(v, k, b)$, and let $\eta=b / k$. Now $B_{1}=(1, \ldots, k)$, without loss of generality let element 1 be introduced 
in $B_{1}$ and let $1^{\prime}$ be changed from $B_{b}$, and suppose that 1 and $1^{\prime}$ are in different columns; let $1^{\prime}$ be in the $s$-th column where $s \neq 1$. Now $\mathcal{B}$ is column-regular and so the column-array of the column-strict representation of each $\mathcal{B}_{i}$ is $A_{1}=[\eta, \ldots, \eta]$.

Now consider the column-strict $\mathcal{B}_{2}=\left\{B_{2}, B_{3}, \ldots, B_{b}, B_{1}\right\}$, where the elements in $B_{2}$ are in the same order as they were in $\mathcal{B}$, element 1 is now in the same column as $1^{\prime}$, the $s$-th column; hence the $s$-th element in the column-array of the column-strict $\mathcal{B}_{2}$ is $\eta+1$, a contradiction. So 1 and $1^{\prime}$ are in the same column in $\mathcal{B}$.

Now consider element $r$ for any fixed $r \in\{2, \ldots, k\}=B_{1} \cap B_{b}$; let it be changed first from $B_{i_{r}}$ (i.e, $r \in B_{1}, \ldots, B_{i_{r}}$ ), and replaced by $r^{\prime}$ in $B_{i_{r}+1}$. See the column-strict arrangement $\mathcal{B}_{i_{r}+1}=\left\{B_{i_{r}+1}, \ldots, B_{b}, B_{1}, \ldots, B_{i_{r}}\right\}$ in Fig. 16(a); elements $r^{\prime}$ and $r$ lie in the same column by the previous argument.

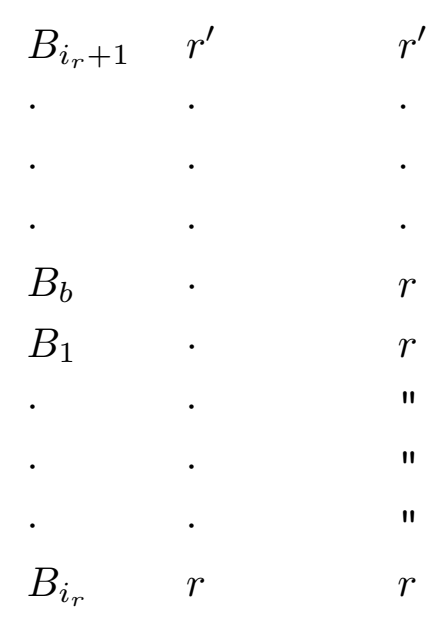

(a)

Fig. 16: Figure for Theorem 11.2.

Now, $r \in B_{b}$ and $r \in B_{1}, \ldots, B_{i_{r}}$, so we have Fig. 16(b). We can retrieve $\mathcal{B}=$ $\left\{B_{1}, \ldots, B_{b}\right\}$ from (b) without changing the columns in which $r$ and $r^{\prime}$ appear. Hence, in $\mathcal{B}$, the $r \in B_{1}$ lies in the same column as the $r \in B_{b}$, and, because $r$ was arbitrarily chosen from $B_{1} \cap B_{b}$, so $\mathcal{B}$ is perfect.

Combining Theorems 11.1 and 11.2 we have the following theorem in which all designs are assumed to be column-strict. 
Theorem 11.3. Let $\mathcal{B}=\left\{B_{1}, \ldots, B_{b}\right\}$ be an $\operatorname{arbitrary} \operatorname{scccd}(v, k, b)$. Then

(i) if $\mathcal{B}$ is perfect the column-array of $\mathcal{B}_{i}$ is a permutation of the column-array of $\mathcal{B}$ for each $i=1, \ldots, b$;

(ii) if $\mathcal{B}$ is column-regular then $\mathcal{B}$ is perfect.

An arbitrary $\operatorname{scccd}(v, k, b), \mathcal{B}$, is element-regular if each of the $v$ elements from $[v]$ is introduced the same number of $\mu=b / v$ times. In the notation of $\S 7$ we have $t_{\mu}=v$.

If $\mathcal{C}$ is tight and element-regular with $\mu=1$ then $v=b=2 k-1$, and so $\mathcal{C}$ is a $\operatorname{sccd}(2 k-1, k, 2 k-1)$ and, by Theorem 6.2 , is isomorphic to $\mathcal{Y}_{k} \in \mathfrak{G}$.

Our final example is shown in Fig. 15(e). It is the representative standardized form of a tight $\operatorname{scccd}(5,2,10)$ which is column-regular with $\eta=5$, and so perfect, and elementregular with $\mu=2$.

Fig. 17 gives some of the numbers of non-isomorphic tight $\operatorname{scccd}(v, k) \operatorname{s}$ for $v \leq 10$.

\section{Fig. 17 here. Please contact the author for the latest version}

Fig. 17: Table showing some of the numbers of non-isomorphic tight $\operatorname{scccd}(v, k) \mathrm{s}$ for $v \geq$ $2 k-2$ and $v \leq 10,(k=2, v \geq 3)$. The number of perfect designs is shown in parenthesis (). For $k \geq 3$ the 1 in column $v=2 k-2$ corresponds to $\mathcal{X}_{k} \in \mathcal{F}$, and for $k \geq 2$ the 1 in column $v=2 k-1$ to $\mathcal{Y}_{k} \in \mathcal{G}$. The symbol - means that $2(k-1) \backslash v(v-1)$ and so a tight design with parameters $(v, k)$ cannot exist. The missing numbers are currently being computed.

Thank you to Donald A. Preece for reading, and commenting upon, an early version of this paper. 


\section{References}

[1] N.C.K. Phillips and W.D. Wallis, Persistent pairs in single-change covering designs, Proceedings of the 24-th Southeastern International Conference on Combinatorics, Graph Theory, and Computing, (Boca Raton FL, 1993). Congressus Numerantium, 96 (1993) 75-82.

[2] D.A. Preece, R.L. Constable, G. Zhang, J.L. Yucas, W.D. Wallis, J.P. McSorley, and N.C.K. Phillips, Tight single-change covering designs, Utilitas Mathematica, 47 (1995) $55-84$.

[3] G.H.J. van Rees, Single-change covering designs II, Congressus Numerantium, 92 (1993) 29-32.

[4] W.D. Wallis, J.L. Yucas, and G.-H. Zhang, Single-change covering designs, Designs, Codes and Cryptography, 3 (1993) 9-19.

[5] G.-H. Zhang, Some new bounds on single-change covering designs, SIAM J. Discrete Math, 7 (1994) 166-171. 